\title{
CANDELS MULTI-WAVELENGTH CATALOGS: SOURCE DETECTION AND PHOTOMETRY IN THE GOODS-SOUTH FIELD
}

\author{
Yicheng Guo $^{1,2}$, Henry C. Ferguson ${ }^{3}$, Mauro Giavalisco ${ }^{2}$, Guillermo Barro $^{1}$, S. P. Willner ${ }^{4}$, Matthew L. N. Ashby ${ }^{4}$, \\ Tomas Dahlen ${ }^{3}$, Jennifer L. Donley $^{5}$, Sandra M. Faber ${ }^{1}$, Adriano Fontana $^{6}$, Audrey Galametz $^{6}$, Andrea Grazian $^{6}$, \\ Kuang-Han Huang ${ }^{3,7}$, Dale D. Kocevski ${ }^{8}$, Anton M. Koekemoer $^{3}$, David C. Koo ${ }^{1}$, Elizabeth J. McGrath ${ }^{9}$, \\ Michael Peth $^{7}$, Mara Salvato ${ }^{10,11}$, Stijn Wuyts ${ }^{10}$, Marco Castellano ${ }^{6}$, Asantha R. Cooray ${ }^{12}$, Mark E. Dickinson ${ }^{13}$, \\ James S. Dunlop ${ }^{14}$, G. G. Fazio ${ }^{4}$, Jonathan P. Gardner ${ }^{15}$, Eric Gawiser ${ }^{16}$, Norman A. Grogin ${ }^{3}$, Nimish P. Hathi ${ }^{17}$, \\ Li-Ting Hsu ${ }^{10}$, Kyoung-Soo Lee $^{18}$, Ray A. Lucas ${ }^{3}$, Bahram Mobasher $^{19}$, Kirpal Nandra ${ }^{10}$, \\ JEFFERY A. NEWMAN ${ }^{20}$, AND ARJEN VAN DER WEL ${ }^{21}$ \\ ${ }^{1}$ UCO/Lick Observatory, Department of Astronomy and Astrophysics, University of California, Santa Cruz, CA, USA; ycguo@ucolick.org \\ ${ }^{2}$ Department of Astronomy, University of Massachusetts, Amherst, MA, USA \\ ${ }^{3}$ Space Telescope Science Institute, Baltimore, MD, USA \\ ${ }^{4}$ Harvard-Smithsonian Center for Astrophysics, Cambridge, MA, USA \\ ${ }^{5}$ Los Alamos National Laboratory, Los Alamos, NM, USA \\ ${ }^{6}$ INAF-Osservatorio di Roma, I-00040 Monteporzio, Italy \\ ${ }^{7}$ Department of Physics and Astronomy, The Johns Hopkins University, Baltimore, MD, USA \\ ${ }^{8}$ Department of Physics and Astronomy, University of Kentucky, Lexington, KY, USA \\ ${ }^{9}$ Department of Physics and Astronomy, Colby College, Waterville, ME, USA \\ ${ }^{10}$ Max-Planck-Institut fur Extraterrestrische Physik, D-85741 Garching bei Munchen, Germany \\ ${ }^{11}$ Excellence Cluster, Boltzmann Strasse 2, D-85748 Garching bei Munchen, Germany \\ ${ }^{12}$ Department of Physics and Astronomy, University of California, Irvine, CA, USA \\ ${ }^{13}$ National Optical Astronomy Observatory, Tucson, AZ, USA \\ ${ }^{14}$ Institute for Astronomy, University of Edinburgh, Royal Observatory, Edinburgh EH9 3HJ, UK \\ 15 NASA's Goddard Space Flight Center, Astrophysics Science Division, Observational Cosmology Laboratory, Greenbelt, MD, USA \\ ${ }^{16}$ Department of Physics and Astronomy, Rutgers University, New Brunswick, NJ, USA \\ ${ }^{17}$ Carnegie Observatories, Pasadena, CA, USA \\ ${ }^{18}$ Department of Physics, Purdue University, West Lafayette, IN, USA \\ ${ }^{19}$ Department of Physics and Astronomy, University of California, Riverside, CA, USA \\ ${ }^{20}$ Department of Physics and Astronomy, University of Pittsburgh, Pittsburgh, PA, USA \\ ${ }^{21}$ Max-Planck Institute for Astronomy, D-69117 Heidelberg, Germany \\ Received 2013 February 7; accepted 2013 June 8; published 2013 July 18
}

\begin{abstract}
We present a UV to mid-infrared multi-wavelength catalog in the CANDELS/GOODS-S field, combining the newly obtained CANDELS HST/WFC3 F105W, F125W, and F160W data with existing public data. The catalog is based on source detection in the WFC3 F160W band. The F160W mosaic includes the data from CANDELS deep and wide observations as well as previous ERS and HUDF09 programs. The mosaic reaches a $5 \sigma$ limiting depth (within an aperture of radius 0.'17) of 27.4, 28.2, and 29.7 AB for CANDELS wide, deep, and HUDF regions, respectively. The catalog contains 34,930 sources with the representative 50\% completeness reaching 25.9 , 26.6, and $28.1 \mathrm{AB}$ in the $\mathrm{F} 160 \mathrm{~W}$ band for the three regions. In addition to WFC3 bands, the catalog also includes data from UV ( $U$ band from both CTIO/MOSAIC and VLT/VIMOS), optical (HST/ACS F435W, F606W, F775W, F814W, and F850LP), and infrared (HST/WFC3 F098M, VLT/ISAAC $K s$, VLT/HAWK-I $K s$, and Spitzer/IRAC 3.6, 4.5, $5.8,8.0 \mu \mathrm{m}$ ) observations. The catalog is validated via stellar colors, comparison with other published catalogs, zero-point offsets determined from the best-fit templates of the spectral energy distribution of spectroscopically observed objects, and the accuracy of photometric redshifts. The catalog is able to detect unreddened star-forming (passive) galaxies with stellar mass of $10^{10} M_{\odot}$ at a $50 \%$ completeness level to $z \sim 3.4(2.8)$, 4.6 (3.2), and 7.0 (4.2) in the three regions. As an example of application, the catalog is used to select both star-forming and passive galaxies at $z \sim 2-4$ via the Balmer break. It is also used to study the color-magnitude diagram of galaxies at $0<z<4$.
\end{abstract}

Key words: catalogs - galaxies: high-redshift - galaxies: photometry - methods: data analysis - techniques: image processing

Online-only material: color figures

\section{INTRODUCTION}

Over the past decade, the Hubble Space Telescope (HST) and modern giant telescopes have opened a new era in observational cosmology. Galaxies are now routinely found in the very early universe, and their evolution can be followed from its early stages to the present. Particularly, deep multi-wavelength imaging surveys have been established as a powerful and efficient tool to push observations up to high redshift and down to faint populations of galaxies. These modern surveys have revealed a complex interplay between galaxy mergers, star formation, and black holes over cosmic time and have led to new insights into the physical processes that drive galaxy formation and evolution.

Of the entire cosmic time, two epochs are particularly of great interest: $z \sim 2$ (cosmic high noon) and $z \sim 8$ (cosmic dawn). In the former, the cosmic star formation history reached its peak (e.g., Hopkins 2004; Hopkins \& Beacom 2006; Bouwens 
et al. 2009), and galaxies quickly assembled their stars (e.g., Daddi et al. 2007; Reddy et al. 2008; Reddy \& Steidel 2009) as well as began to differentiate into morphological sequence (e.g., Cassata et al. 2008; Kriek et al. 2009; Whitaker et al. 2011; Wang et al. 2012). In the latter, detection and physical properties of galaxies place essential constraints on the reionization (e.g., Fontana et al. 2010; Oesch et al. 2010; Grazian et al. 2011; Bouwens et al. 2012; Finkelstein et al. 2012a), the accretion history of galaxies at early times (e.g., Bouwens et al. 2010a; Finkelstein et al. 2010; McLure et al. 2010; Trenti et al. 2011; Yan et al. 2012), as well as the formation of metals and dust in the intergalactic medium (e.g., Bouwens et al. 2010b; Dunlop et al. 2012; Finkelstein et al. 2012b). Because two important spectral features, namely, the Balmer break at $z \sim 2$ and the Lyman break at $z \sim 8$, are redshifted to near-infrared (NIR) bands at the two epochs, a NIR survey with deep sensitivity and high spatial resolution is required for robust investigation of galaxies during these epochs. Incorporating these deep NIR data into a multiwavelength catalog would significantly improve measurements of galaxy properties at $z>2$.

The Cosmic Assembly Near-infrared Deep Extragalactic Legacy Survey (CANDELS; Grogin et al. 2011; Koekemoer et al. 2011) is designed to document galaxy formation and evolution over the redshift range of $z=1.5-8$. The core of CANDELS is to use the revolutionary near-infrared HST/WFC3 camera, installed on HST in 2009 May, to obtain deep imaging of faint and faraway objects. CANDELS also uses HST/ACS to carry out parallel observations, aiming to providing unprecedented panchromatic coverage of galaxies from optical wavelengths to the near-IR. Covering approximately $800 \mathrm{arcmin}^{2}$, CANDELS will image over 250,000 distant galaxies within five popular sky regions which possess rich existing data from multiple telescopes and instruments: GOODS-S, GOODS-N, UDS, EGS, and COSMOS. The strategy of five widely separated fields mitigates cosmic variance and yields statistically robust and complete samples of galaxies down to a stellar mass of $10^{9} M_{\odot}$ to $z \sim 2$, and around the knee of the ultraviolet luminosity function of galaxies to $z \sim 8$. It will also find and measure Type Ia supernovae at $z>1.5$ to test their accuracy as standard candles for cosmology.

The GOODS-S field, centered at $\alpha(\mathrm{J} 2000)=03^{\mathrm{h}} 32^{\mathrm{m}} 30^{\mathrm{s}}$ and $\delta(\mathrm{J} 2000)=-27^{\mathrm{d}} 48^{\mathrm{m}} 20^{\mathrm{s}}$ and located within the Chandra Deep Field South (CDFS; Giacconi et al. 2002), is a sky region of about $170 \operatorname{arcmin}^{2}$ which has been targeted for some of the deepest observations ever taken by NASA's Great Observatories, HST, Spitzer, and Chandra as well as by other world-class telescopes. The field has been imaged in the optical wavelength with HST/ACS in F435W, F606W, F775W, and F850LP bands as part of the HST Treasury Program: the Great Observatories Origins Deep Survey (GOODS; Giavalisco et al. 2004); in the mid-IR (3.6-24 $\mu \mathrm{m})$ wavelength with Spitzer as part of the GOODS Spitzer Legacy Program (PI: M. Dickinson); and in the X-ray with the deepest Chandra sky survey ever taken, with a total integration time of $4 \mathrm{Ms}$ (Xue et al. 2011). The field also has very deep far-IR to submillimeter data from Herschel Space Observatory from the PEP survey (Lutz et al. 2011) and the HerMes survey (Oliver et al. 2012), deep submillimeter (1.1 mm) observation with AzTEC/ASTE (Scott et al. 2010), and radio imaging from the VLA (Kellermann et al. 2008), ATCA (Norris et al. 2006; Zinn et al. 2012), and VLBA (Middelberg et al. 2011). Furthermore, the field has been the subject of numerous spectroscopic surveys (e.g., Le Fèvre et al. 2004; Szokoly et al. 2004; Mignoli et al. 2005; Cimatti et al.
2008; Vanzella et al. 2008; Popesso et al. 2009; Balestra et al. 2010; Silverman et al. 2010). These rich and deep existing data, covering the full wavelength range, make GOODS-S one of the best fields to study galaxy formation and evolution.

This paper presents a multi-wavelength catalog of GOODS-S based on CANDELS WFC3 F160W detection, combining both CANDELS data and available public imaging data from UV to mid-IR wavelengths. The catalog includes $U$-band images obtained with the CTIO Blanco telescope and with the Visible Multi-Object Spectrograph (VIMOS) on the Very Large Telescope (VLT), HST/ACS images in the F435W, F606W, F775W, F814W, and F850LP bands, HST/WFC3 images in the F098M, F105W, F125W, and F160W bands, $K s$-band image with the Infrared Spectrometer and Array Camera (ISAAC) and the High Acuity Wide field $K$-band Imager (HAWK-I) on the VLT, as well as Spitzer images at 3.5, 4.5, 5.8, and $8.0 \mu \mathrm{m}$.

The paper is organized as follows: Section 2 briefly summarizes the data sets included in our catalog. Section 3 discusses source detection in the CANDELS F160W image and photometry measurement on HST images. Section 4 discusses photometry measurements on low-resolution images. Section 5 evaluates the quality of our photometry catalog. Section 6 presents a few simple applications of our catalog to study galaxy formation and evolution. The summary is given in Section 7.

All magnitudes in the paper are on the AB scale (Oke 1974) unless otherwise noted. We adopt a flat $\Lambda \mathrm{CDM}$ cosmology with $\Omega_{m}=0.3, \Omega_{\Lambda}=0.7$ and use the Hubble constant in terms of $h \equiv H_{0} / 100 \mathrm{~km} \mathrm{~s}^{-1} \mathrm{Mpc}^{-1}=0.70$.

The CANDELS GOODS-S multi-wavelength catalog and its associated files-all SExtractor photometry of the HST bands as well as the system throughput of all included filters-are made publicly available on the CANDELS Web site, ${ }^{22}$ in the Mikulski Archive for Space Telescopes (MAST), ${ }^{23}$ via the online version of the article, and the Centre de Donnees astronomiques de Strasbourg (CDS). They are also available in the Rainbow Database $^{24}$ (Pérez-González et al. 2008; Barro et al. 2011), which features a query menu that allows users to search for individual galaxies, create subsets of the complete sample based on different criteria, and inspect cutouts of the galaxies in any of the available bands. It also includes a cross-matching tool to compare against user uploaded catalogs.

\section{DATA}

Table 1 summarizes the multi-wavelength data sets included in our catalog. The table lists the effective wavelength, the FWHM of the filter, the FWHM of the point-spread function (PSF), and the limiting magnitude of each band in our catalog. For each band, we calculate the $5 \sigma$ limiting magnitude with an aperture whose radius is equal to the FWHM of the band. No aperture correction is applied to the limiting magnitude. Transmission curves of all filters used in our catalog are plotted in Figure 1. The sky coverage of observations used in our catalog is shown in Figure 2.

\subsection{HST/WFC3 Imaging}

Several programs have carried out observations with $H S T / W F C 3$ IR channels in GOODS-S, including CANDELS, the HST/WFC3 Early Release Science (ERS; Windhorst et al.

\footnotetext{
$22 \mathrm{http} / / /$ candels.ucolick.org

$23 \mathrm{http}: / /$ archive.stsci.edu

24 US: https://arcoiris.ucolick.org/Rainbow_navigator_public; and Europe: https://rainbowx.fis.ucm.es/Rainbow_navigator_public.
} 


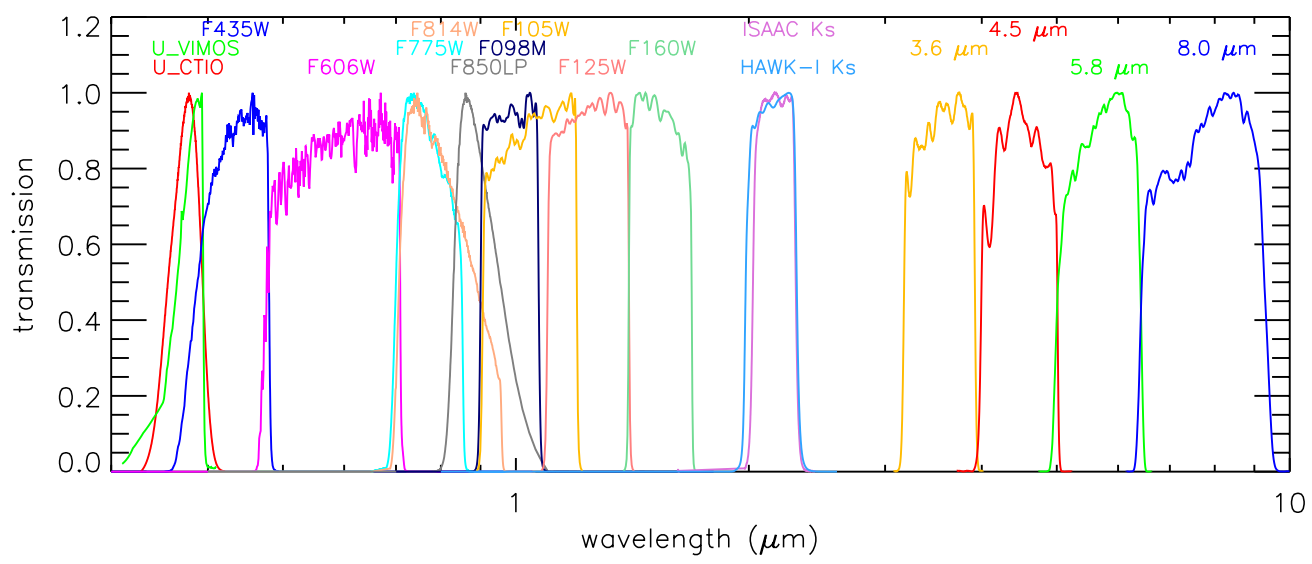

Figure 1. Transmission of all filters used in our multi-wavelength catalog in the CANDELS GOODS-S field. All transmissions are normalized to have a maximum value of unity.

(A color version of this figure is available in the online journal.)

Table 1

Summary of Data Included in the CANDELS GOODS-S Catalog

\begin{tabular}{|c|c|c|c|c|c|}
\hline Instrument & Filter & $\begin{array}{c}\text { Effective } \\
\text { Wavelength }^{\mathrm{a}} \\
(\AA)\end{array}$ & $\begin{array}{c}\text { Filter } \\
\text { FWHM } \\
(\AA)\end{array}$ & $\begin{array}{c}\text { PSF } \\
\text { FWHM } \\
(\operatorname{arcsec})\end{array}$ & $\begin{array}{c}5 \sigma \text { Limiting } \\
\text { Depth } \\
(\mathrm{AB})^{\mathrm{b}}\end{array}$ \\
\hline Blanco/MOSAIC II & U_CTIO & 3734 & 387 & 1.37 & 26.63 \\
\hline VLT/VIMOS & U_VIMOS & 3722 & 297 & 0.80 & 27.97 \\
\hline \multirow[t]{5}{*}{$H S T / \mathrm{ACS}$} & F435W & 4317 & 920 & 0.08 & $28.95 / 30.55^{\mathrm{c}}$ \\
\hline & F606W & 5918 & 2324 & 0.08 & $29.35 / 31.05^{\mathrm{c}}$ \\
\hline & F775W & 7693 & 1511 & 0.08 & $28.55 / 30.85^{\mathrm{c}}$ \\
\hline & F814W & 8047 & 1826 & 0.09 & 28.84 \\
\hline & F850LP & 9055 & 1236 & 0.09 & $28.55 / 30.25^{\mathrm{c}}$ \\
\hline \multirow[t]{4}{*}{$H S T /$ WFC3 } & F098M & 9851 & 1696 & 0.13 & 28.77 \\
\hline & F105W & 10550 & 2916 & 0.15 & $27.45 / 28.45 / 29.45^{\mathrm{d}}$ \\
\hline & F125W & 12486 & 3005 & 0.16 & $27.66 / 28.34 / 29.78^{d}$ \\
\hline & F160W & 15370 & 2874 & 0.17 & $27.36 / 28.16 / 29.74^{\mathrm{d}}$ \\
\hline VLT/ISAAC & $K s$ & 21605 & 2746 & $0.48_{-0.1}^{+0.1 \mathrm{e}}$ & $25.09_{-0.32}^{+0.60 \mathrm{e}}$ \\
\hline VLT/HAWK-I & $K s$ & 21463 & 3250 & $\sim 0.4$ & 26.45 \\
\hline \multirow[t]{4}{*}{ Spitzer/IRAC } & $3.6 \mu \mathrm{m}$ & 35508 & 7432 & 1.66 & $26.52^{f}$ \\
\hline & $4.5 \mu \mathrm{m}$ & 44960 & 10097 & 1.72 & $26.25^{\mathrm{f}}$ \\
\hline & $5.8 \mu \mathrm{m}$ & 57245 & 13912 & 1.88 & $23.75^{\mathrm{g}}$ \\
\hline & $8.0 \mu \mathrm{m}$ & 78840 & 28312 & 1.98 & $23.72^{\mathrm{g}}$ \\
\hline
\end{tabular}

Notes.

${ }^{\text {a }}$ Effective wavelength is calculated as $\lambda_{\text {eff }}=\sqrt{\left(\int S(\lambda) \lambda d \lambda\right) /\left(\int S(\lambda) \lambda^{-1} d \lambda\right)}$ (Tokunaga \& Vacca 2005).

b Aperture magnitude at $5 \sigma$ within $r_{\text {ap }}=1$ FWHM of the PSF. Point-source magnitude will be brighter than this by the aperture correction assuming the profile of the PSF. Aperture corrections for extended sources will depend on their surface brightness profiles.

c The limiting depths of ACS bands are measured within apertures with a fixed radius of $00^{\prime \prime} 09$. Each band has two measurements: one for GOODS-S V2.0 and the other for HUDF.

${ }^{\mathrm{d}}$ The limiting depths of WFC 3 bands are measured within apertures with a fixed radius of 0 '! 17 . Each band has three measurements: for CANDELS-wide, CANDELS-deep, and HUDF09, respectively.

${ }^{\mathrm{e}}$ PSF and depth vary among ISAAC tiles. We show the median and the 15 and 85 percentiles of all available tiles.

${ }^{\mathrm{f}}$ This is the depth of the SEDS GOODS-S region measured through apertures. For the SEDS non-GOODS region, the $5 \sigma$ depth is $\sim 25.4 \mathrm{AB}$ mag measured through photometry of artificial sources. Ashby et al. (2013) give details on the depth of SEDS depths.

$\mathrm{g}$ This is the average depth of the two GOODS Spitzer epochs. The overlapped region, which is in the CANDELS-deep region, is deeper by about 0.35 mag.

2011), and HUDF09 (Bouwens et al. 2010a). To maximize the scientific merit of our multi-wavelength catalog, we combine all published $H S T /$ WFC3 imaging within GOODS-S prior to 2012 June to make a "max-depth" co-added mosaic of HST/WFC3 images. These co-added images provide each source the deepest NIR observation to date to ensure the least uncertainty when these bands are used for measuring photometric redshifts and stellar masses. A potential drawback is the inhomogeneity of the depth across the whole GOODS-S field, which may bring complexity when measuring the completeness of the catalog. However, even within each sub-region of the field, for example the CANDELS wide region, the actual point-by-point exposure times and sensitivities vary considerably. Therefore, the inhomogeneity issue exists anyway.

CANDELS observed GOODS-S with the HST/WFC3 F105W, F125W, and F160W filters using two strategies: deep and wide. The deep region covers the middle one-third of the GOODS-S ACS region with an area of $\sim 55 \operatorname{arcmin}^{2}$ by three, 


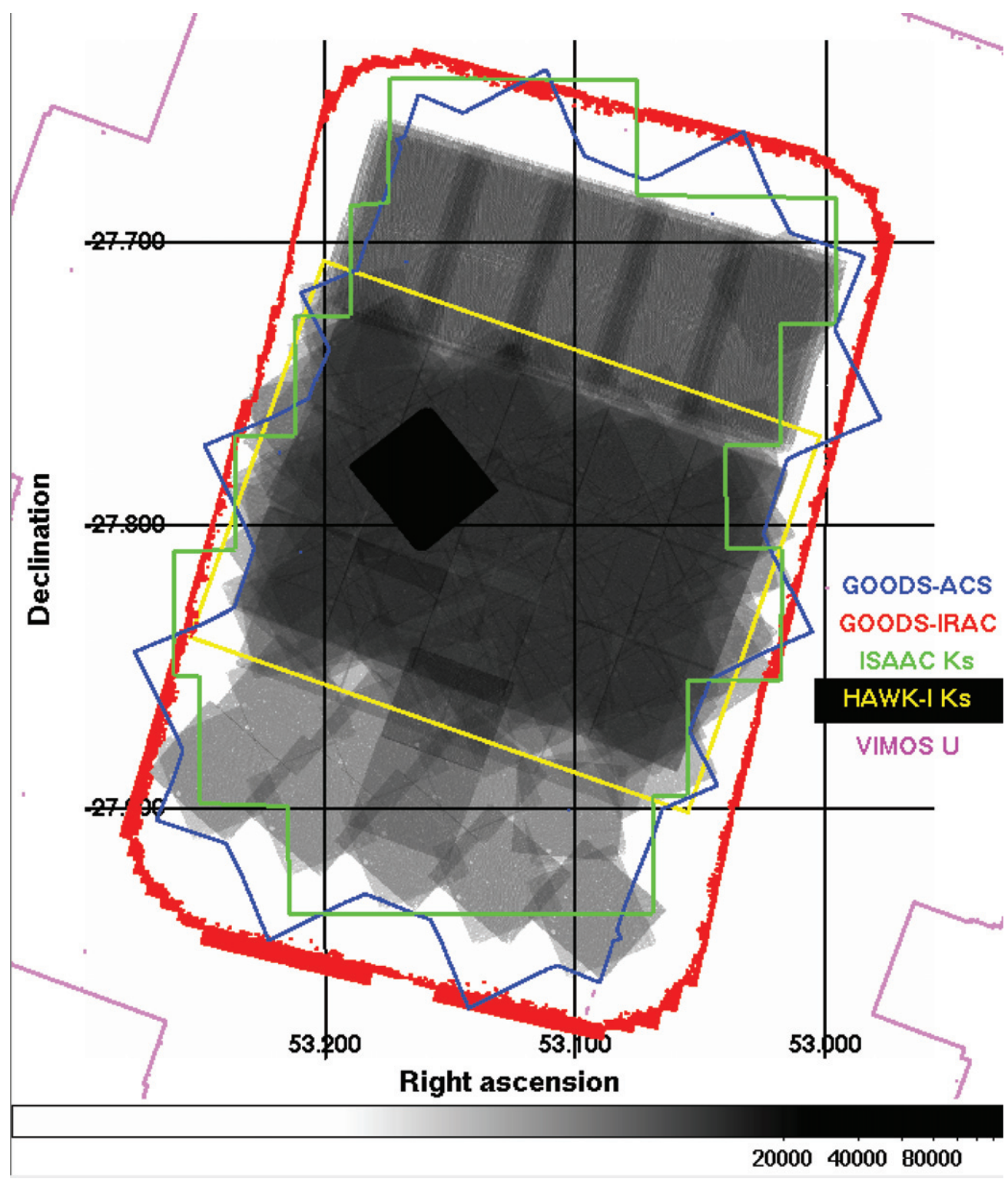

Figure 2. Sky coverage of data sets used in our catalog. The gray scale shows the exposure time of our max-depth F160 mosaic, which includes the CANDELS wide and deep region, ERS, and HUDF09. Coverage of ancillary data from UV to MIR is also shown: VLT/VIMOS U (magenta), GOODS HST/ACS (blue), VLT/ISAAC $K s$ (green), VLT/HAWK-I Ks (yellow), and GOODS Spitzer/IRAC (red). The entire field is covered by both SEDS Spitzer/IRAC and VLT/VIMOS $U$.

four, and six orbits with the F105W, F125W, and F160W filters, respectively. The wide region covers the southern one-third of the GOODS-S ACS region and has approximately one-orbit exposure for all three bands. Grogin et al. (2011) and Koekemoer et al. (2011) give details of CANDELS HST/WFC3 observations, survey design, and data reduction. The GOODS-S region was also observed by CANDELS with the HST/ACS F814W filter in parallel.

The northern one-third of the GOODS-S region was observed by HST/WFC3 ERS (Windhorst et al. 2011) with the F098M, F125W, and F160W filters. ERS covered this region with two orbits in each of the three bands.

An area of about $4.6 \mathrm{arcmin}^{2}$ in GOODS-S, the Hubble Ultra Deep Field (HUDF), is covered by ultra-deep HST/WFC3 imaging from G. Illingworth's HUDF09 program (GO 11563; Bouwens et al. 2010a). HUDF09 observed the field for 24, 34 , and 53 orbits in the F105W, F125W, and F160W bands. The CANDELS deep/wide region and ERS, together with the HUDFs, create a three-tiered "wedding-cake" approach that has proven efficient for extragalactic surveys. We carry out our own data reduction on both ERS and HUDF images and drizzled them to $0^{\prime \prime} .06$ pixel $^{-1}$ to match our CANDELS pixel scale (see Koekemoer et al. 2011 for details). The distributions of exposure time and limiting magnitude of our max-depth F160W mosaic are shown in Figure 3.

\subsection{HST/ACS Imaging}

The HST/ACS images used in our catalog are version v3.0 of the mosaicked images from the GOODS HST/ACS Treasury Program. They consist of data acquired prior to the HST Servicing Mission 4, including mainly data of the original GOODS HST/ACS program in HST Cycle 11 (GO 9425 and 9583; see Giavalisco et al. 2004) and additional data acquired on the GOODS fields during the search for high-redshift Type Ia supernovae carried out during Cycles 12 and 13 (Program ID 9727; PI: Saul Perlmutter; and 9728, 10339, 10340; PI: Adam Riess; see, e.g., Riess et al. 2007). The GOODS-S field was observed in the Advanced Camera for Surveys (ACS) $B, V, i$, 


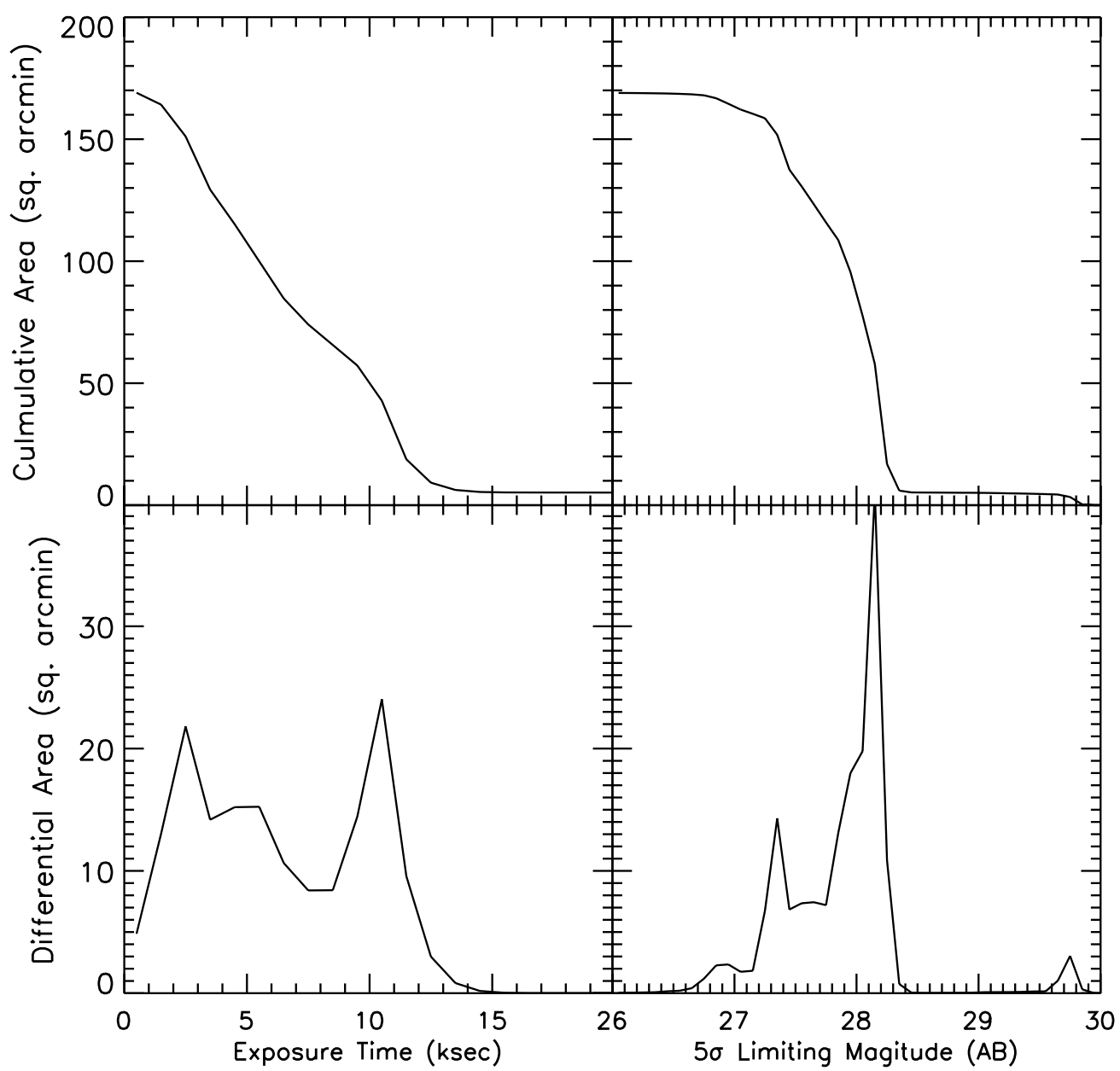

Figure 3. Distributions of exposure time and limiting magnitude of our max-depth F160W mosaic used as the detection image of our catalog. The left column shows the cumulative (upper panel) and differential (lower) distributions of the exposure time, while the right column shows the same distributions of the $5 \sigma$ limiting magnitude of the image. HUDF09 is not shown in the left column as its exposure time $(\sim 155 \mathrm{ks})$ is far off-axis.

and $z$ bands with total exposure time of $7200,5450,7028$, and $18,232 \mathrm{~s}$.

\subsection{Ground-based Imaging}

GOODS-S is covered by a large number of ground-based images. Combined into our catalog is imaging of the CTIO $U$ band, VLT/VIMOS $U$ band, VLT/ISAAC $K s$ band, and VLT/HAWK-I $K s$ band.

The CDF-S/GOODS field was observed by the MOSAIC II imager on the CTIO $4 \mathrm{~m}$ Blanco telescope to obtain deep $U$-band observations in 2001 September. The observations were taken through CTIO filter c6021, the SDSS $u^{\prime}$ filter. ${ }^{25}$ This filter was chosen in order to minimize bandpass overlap with the standard $B$ band and the ACS F435W filter in the hopes of providing better photometric redshift and Lyman break constraints on $z \sim 3$ galaxies. The final image has a total integration time of about $17.5 \mathrm{hr}$.

Another $U$-band survey in GOODS-S was carried out using the VIMOS instrument mounted at the Melipal Unit Telescope of the VLT at ESO's Cerro Paranal Observatory, Chile. This large program of ESO (168.A-0485; PI: C. Cesarsky) was obtained in service mode observations in UT3

25 A red leak is found in the filter, between 7000 and $11000 \AA$. The red leak affects the photometry of objects with red colors. Smith et al. (2002) found that the red leak makes objects with red color $\left(u^{\prime}-i^{\prime}>5 \mathrm{mag}\right)$ brighter in the $u^{\prime}$ band than they should be. between 2004 August and fall 2006, with a total time allocation of $40 \mathrm{hr}$. Nonino et al. (2009) give details of the $U$-band imaging.

In the ground-based NIR, imaging observations of the CDFS were carried out in $J, H, K s$ bands using the ISAAC instrument mounted at the Antu Unit Telescope of the VLT. Data were obtained as part of the ESO Large Programme 168.A-0485 (PI: C. Cesarsky) as well as ESO Programmes 64.O-0643, 66.A-0572, and 68.A-0544 (PI: E. Giallongo) with a total allocation time of $\sim 500 \mathrm{hr}$ from 1999 October to 2007 January. The data cover 172.4, 159.6, and $173.1 \operatorname{arcmin}^{2}$ of the GOODS/CDF-S region in $J, H$, and $K s$, respectively. Because the CANDELS HST/WFC3 F125W and F160W bands surpass the ground-based $J$ and $H$ bands in both spatial resolution and sensitivity, we use only the $K s$ data from the GOODS VLT/ISAAC program. Retzlaff et al. (2010) give details on the ISAAC $K s$-band imaging.

The CANDELS/GOODS-S field was also observed in the NIR as part of the ongoing HAWK-I UDS and GOODS-S survey (HUGS; VLT large program ID 186.A-0898; PI: A. Fontana; A. Fontana et al., in preparation) using the High Acuity Wide field $K$-band Imager (HAWK-I) on VLT. Included in this paper are the two deep HAWK-I pointings in the CANDELS deep region. Each pointing was imaged in the $K s$ band for $\sim 31 \mathrm{hr}$. A. Fontana et al. (in preparation) will give more details on the HUGS survey and data. 


\subsection{Spitzer/IRAC Imaging}

GOODS-S was observed by Spitzer/IRAC (Fazio et al. 2004) with four channels $(3.6,4.5,5.8$, and $8.0 \mu \mathrm{m})$ in two epochs with a separation of six months (2004 February and 2004 August) by the GOODS Spitzer Legacy project (PI: M. Dickinson). Each epoch contained two pointings, each with total extent approximately 10 arcmin on a side. IRAC observed simultaneously in all four channels, with channels 1 and 3 (3.6 and $5.8 \mu \mathrm{m})$ covering one pointing on the sky and channels 2 and $4(4.5$ and $8.0 \mu \mathrm{m})$ covering another pointing. After two epochs, GOODS-S has complete coverage in all four IRAC channels with an overlap strip in the middle receiving twice the exposure time of the rest of the field. The exposure time per channel per sky pointing was approximately $25 \mathrm{hr}$ per epoch and doubled in the overlap strip. We include the GOODS 5.8 and $8.0 \mu \mathrm{m}$ imaging in our catalog.

The Spitzer/IRAC 3.6 and $4.5 \mu \mathrm{m}$ images used here were drawn from the Spitzer Extended Deep Survey (SEDS; PI: G. Fazio; Ashby et al. 2013). The SEDS ECDFS observations were acquired during three separate visits made during the warm Spitzer mission. Each SEDS visit covered a region surrounding the existing IRAC exposures from the GOODS program, and the SEDS mosaics incorporate the pre-existing cryogenic observations. The SEDS mosaics were pixellated to 0 .'6 and were constructed with a tangent-plane projection designed to match that of the CANDELS HST/WFC3 mosaics. Total $3 \sigma$ depths in the SEDS-only portions of the field are $\sim 26 \mathrm{AB}$ mag measured through photometry of artificial sources. Catalogs and source counts in the 3.6 and $4.5 \mu \mathrm{m}$ mosaics are fully described by Ashby et al. (2013).

\section{PHOTOMETRY OF HST IMAGES}

\subsection{Source Detection in Max-depth F160W Image}

The scientific goals of CANDELS extend from studying the morphology of galaxies at the cosmic high noon $(z \sim$ 2) to searching for faint galaxies at the cosmic dawn $(z>$ 7). Therefore, our source detection strategy must be efficient in detecting both bright/large and faint/small sources. It is, however, almost impossible to design a single set of SExtractor parameters to achieve this goal. A mild detection and deblending threshold avoids breaking large/bright sources into small pieces but misses a large fraction of faint sources. On the other hand, an aggressive detection threshold able to detect very faint sources overdeblends bright/large galaxies. To ensure that our catalog provides reliable detection for both the bright/large and faint/small sources, we adopt a two-mode detection strategy, which has been shown to be successful and efficient in other deep sky surveys, e.g., GEMS (Rix et al. 2004) and STAGES (Gray et al. 2009).

We run our modified SExtractor (see Galametz et al. 2013) on our co-added max-depth version of HST/WFC3 F160W image to detect sources in two modes: "cold" and "hot." In the cold mode, the SExtractor configuration is designed to detect bright/large sources without overdeblending them. In the hot mode, the SExtractor configuration is pushed to detect faint sources toward the limiting depth of the image. In this mode, bright/large sources may be dissolved, and some of their substructures are treated as independent sources. We then follow the strategy of GALAPAGOS (Barden et al. 2012) to merge the two modes: all cold sources are kept in the final detection catalog, but hot sources within the Kron radius of any cold sources are treated as substructures of the cold sources and
Table 2

SExtractor Parameters in Cold and Hot Modes

\begin{tabular}{lcc}
\hline \hline & Cold Mode & Hot Mode \\
\hline DETECT_MINAREA & 5.0 & 10.0 \\
DETECT_THRESH & 0.75 & 0.7 \\
ANALYSIS_THRESH & 5.0 & 0.8 \\
FILTER_NAME & tophat_9.0_9x9.conv & gauss_4.0_7x7.conv \\
DEBLEND_NTHRESH & 16 & 64 \\
DEBLEND_MINCONT & 0.0001 & 0.001 \\
BACK_SIZE & 256 & 128 \\
BACK_FILTERSIZE & 9 & 5 \\
BACKPHOTO_THICK & 100 & 48 \\
MEMORY_OBJSTACK & 4000 & 4000 \\
MEMORY_PIXSTACK & 400000 & 5000 \\
MEMORY_BUFSIZE & 5000 & \\
\hline
\end{tabular}

excluded from the final catalog. Only the hot sources that are not within the Kron radius of any cold sources are included in the final catalog. We refer readers to GALAPAGOS (Barden et al. 2012) and Galametz et al. (2013) for details of this twomode strategy and its application on CANDELS images. In total, we detect 34,930 sources from our max-depth F160W mosaic. Among them, 26,835 sources are detected by the cold mode and 8095 sources by the hot mode. The key SExtractor parameters used in our source detection are listed in Table 2.

The inhomogeneous depth across our detection image brings a new issue on the detection and deblending threshold. Ideally, in order to construct a catalog whose completeness is similar over regions with various depths, one would use a distinct configuration for each region or use a fixed surface brightness threshold instead of signal-to-noise ratio $(\mathrm{S} / \mathrm{N})$ to detect sources. In our catalog, however, we use the same $\mathrm{S} / \mathrm{N}$ threshold for detecting sources in all regions. The choice is based on two facts. First, the purpose of our max-depth catalog is to push our detection ability in each region to its limit. Therefore, it is the limit on the faint end instead of a homogeneous completeness that we are interested in. Second, even within one region, the depth varies from point to point due to the observing pattern. For example, in the "wide" region, the exposure time of points observed by more than one tile could be $3-4$ times higher than that of points observed only once. Because the inhomogeneity exists not only between different regions but also within each region, it would be impossible to design a distinct detection configuration for each region. For readers who are interested in completeness, the catalog provides the exposure time (or depth) of each source, which can be used to construct a sample that is complete to the same depth over all regions.

The differential number densities of sources detected in the three (wide, deep + ERS, and HUDF) regions are shown in Figure 4. We also separate the contributions of sources detected in the cold and hot modes. In all regions, the distribution peak of the hot-detected sources is almost 1-1.5 mag fainter than that of the cold-detected ones. The hot mode significantly improves our detection ability toward the limiting depth of our images. To make a fair comparison between the total magnitude (MAG_AUTO) of detected sources and the limiting depth, we correct the $5 \sigma$ limiting depths of the F160W band in Table 1, which is measured within an aperture, to include the light outside the aperture by assuming the light profile of the F160W PSF. The peak of the hot detected sources matches the corrected depth (purple line) very well in all regions. Compared to the cold mode, the hot mode boosts the source number density at the limiting depth by a factor of $\sim 20$. 


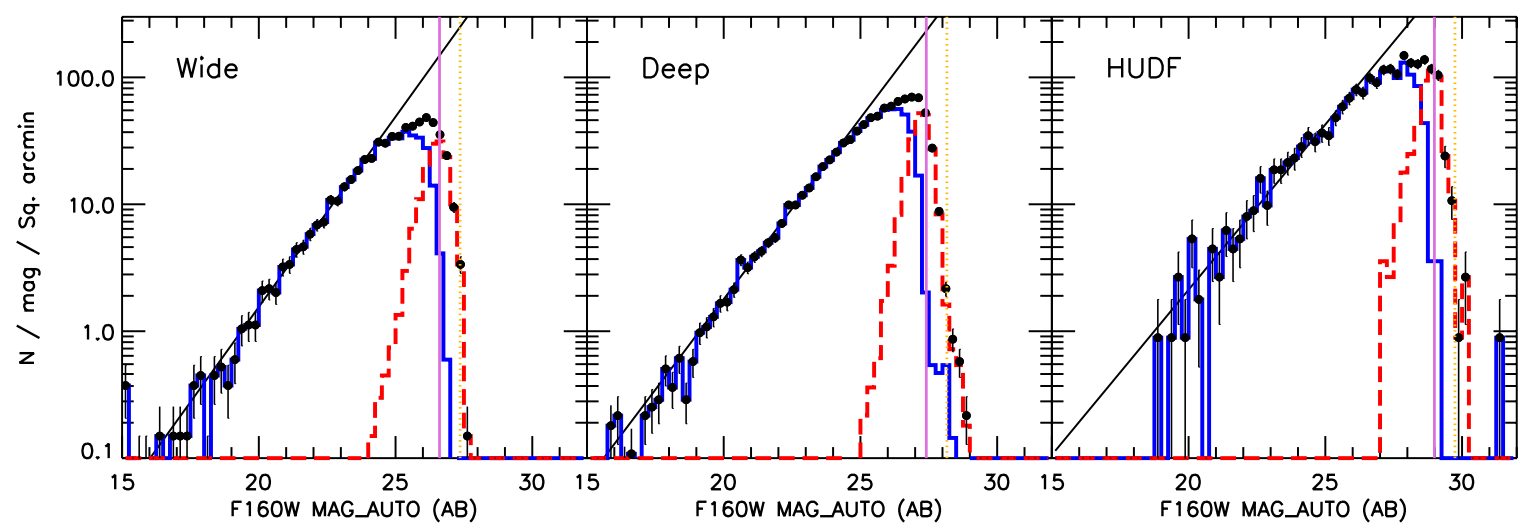

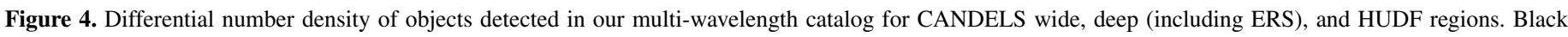

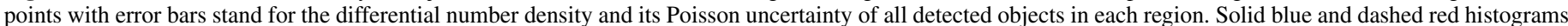

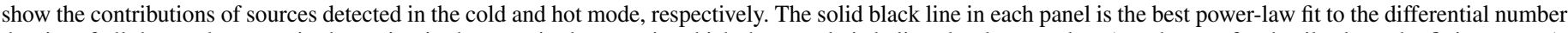

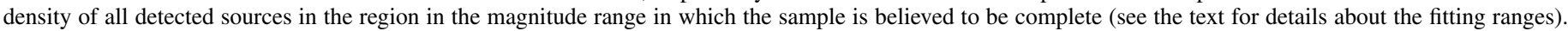

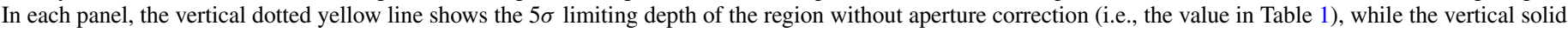
purple line shows the depth after point-source aperture correction.

(A color version of this figure is available in the online journal.)

The differential number density provides a rough way to estimate the completeness of our catalog in different regions. We fit a power law to the differential number density of each region. The catalog probably becomes incomplete when the actual differential number density significantly deviates from the best-fit power law at the faint end. We only fit the power law in the magnitude range in which the catalog is believed to be complete as well as has good number statistics: 20-24 mag, 20-24 mag, and 21-26 mag for the wide, deep, and HUDF regions, respectively. The actual differential number density becomes lower than the best-fit power law by a factor of two at 25.9, 26.6, and $28.1 \mathrm{mag}$ for the three regions. Therefore, our catalog is $\sim 50 \%$ complete at these magnitudes for the three regions.

This completeness is only a rough estimate. It depends on the assumption that our catalog is complete in the fitting range. What is more, it assumes that the completeness only depends on the flux of sources. It also assumes the counts are a power law over the magnitude range that was fit, and that this power law extends to fainter magnitudes. In fact, the completeness depends on the flux, size, and even morphology of sources. The completeness estimated here is just an overall value for objects with mixed types and should be used with caution when dealing with a specific type of object.

In order to accurately estimate the completeness of sources with various fluxes, sizes, and light profiles, we carry out Monte Carlo simulations of detecting fake sources with the same detection strategy used in our catalog for each region. The fake galaxies populate the magnitude range of $20 \mathrm{mag}<$ F160W $<30$ mag and the range of galaxy half-light radii from 0.1 pixel to 30 pixels. The input galaxies are spheroids with a de Vaucouleurs surface brightness profile (Sérsic index $n=4$ ) and disks with an exponential profile $(n=1)$. The simulated galaxies are convolved with the F160W PSF and inserted into our F160W mosaic with additional Poisson noise. We use the SExtractor parameters of both our cold and hot modes to recover the fake sources. The resulting completeness limits in a plane of magnitude and half-light radius are shown in Figure 5.

In this figure, we overplot the power-law-estimated 50\% completeness at the median size (the half-light radius measured by SExtractor) of sources in each region. These values are in good agreement with those measured by the simulation for sources with the input size similar to the median of the actual size distribution. The 20th and 80th percentiles of the actual size distributions span a fairly small range of about $0.3 \mathrm{dex}$, within which the $50 \%$ completeness measured through the simulation changes mildly for each region. Therefore, the 50\% completeness estimated from the power law can be used as a representative limit for the majority of sources in our catalog. For sources with much larger sizes, however, their completeness is much worse than this representative value. The completeness also depends on the morphology of sources. Sources with higher light concentration $(n=4)$ are complete to fainter magnitude than those with lower concentration $(n=1)$ at the same radius.

\subsection{Photometry of Other HST Images}

Photometry of other HST bands, namely, ACS F435W, F606W, F775W, F814W, F850LP and WFC3 F098M, F105W, and $\mathrm{F} 125 \mathrm{~W}$, is measured by running SExtractor in dual-image mode with our WFC3 F160W mosaic as the detection image. All these bands are smoothed by using the IRAF/PSFMATCH package $^{26}$ to match their PSFs to that of the F160W image. The WFC3 PSFs are generated by combining a core from TinyTim and wing from stacked stars (see van der Wel et al. 2012 for details), while the ACS PSFs are generated by stacking stars. SExtractor is run on the PSF-matched image of each band in both cold and hot modes with the same configurations as used for the F160W photometry. Therefore, the source detection, segmentation area, and isophotal area of sources are identical for the F160W and other HST images. The cold and hot photometry of each band is merged based on the F160W cold and hot detection and combination.

Because the isophotal areas of a source in all HST bands are identical, the isophotal fluxes (FLUX_ISO in SExtractor) can be used to measure colors among the HST bands. Under some circumstances, e.g., measuring stellar mass from spectral energy distribution (SED) fitting, the total fluxes of all bands are needed. For these purposes, we provide an inferred

\footnotetext{
26 We set the PSFMATCH parameters "filter" = "replace" and "threshold" = 0.01 to replace the very high frequency and low signal-to-noise components of the PSF matching function with a model computed from the low-frequency and high signal-to-noise components of the matching function.
} 


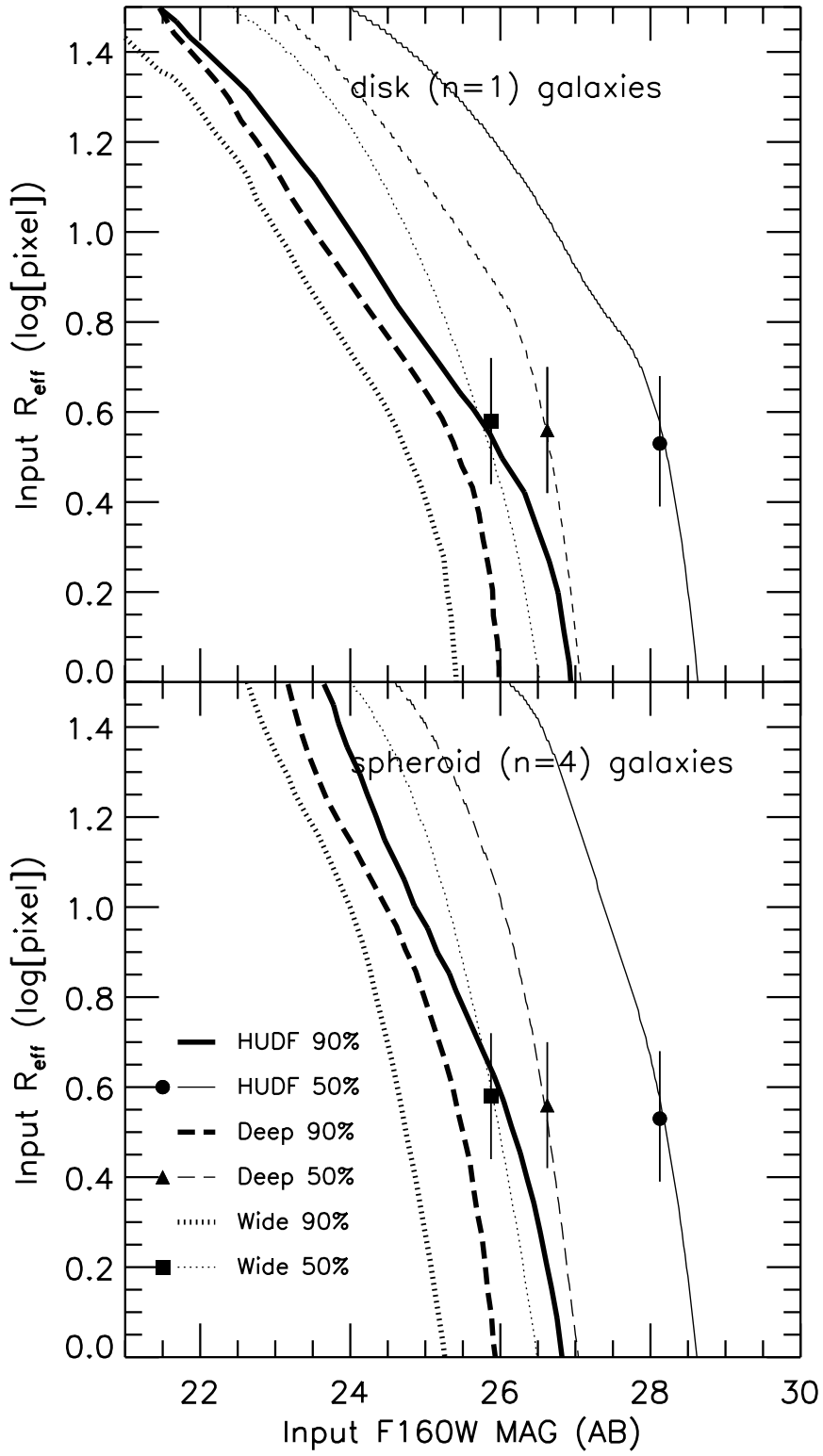

Figure 5. Completeness estimated from detecting fake sources from our F160W mosaics. The light profile of fake sources is assumed to be an exponential disk (Sérsic index $n=1$, top panel) or a de Vaucouleurs profile ( $n=4$, bottom panel). In each case, the constant curves of $50 \%$ and $90 \%$ completeness of each region are plotted in the input size-magnitude diagram, as the labels show. The 50\% completeness estimated through the best-fit power law to the actual differential number density (Figure 4) is also plotted (symbols with error bars) as a reference. The symbols are placed at the median of the size (half-light radius measured by SExtractor) distribution of each region, and their error bars stand for the 20th and 80th percentile of the size distribution.

total flux for each band in the following way. For the detection band $(\mathrm{F} 160 \mathrm{~W})$, we use the photometry from within the Kron elliptical aperture (FLUX_AUTO in SExtractor) as the measure of total flux. We then derive an aperture correction factor, apcorr $\equiv$ FLUX_AUTO/FLUX_ISO, and apply it to other HST bands to convert their isophotal fluxes and uncertainties into the total fluxes and uncertainties: FLUX_TOTAL = apcorr $\times$ FLUX_ISO and FLUXERR_TOTAL $=$ apcorr $\times$ FLUXERR_ISO. Our aperture correction method provides an accurate estimate of colors and fluxes subject to the prior assumption that the PSF-convolved profile is the same in all bands. While this assumption is not likely to hold for well-resolved sources, most of the sources in the image are small enough that any wavelength dependence of the galaxy profile will have very little impact on the integrated flux ratio. The total flux and its uncertainty (FLUX_TOTAL and FLUXERR_TOTAL) are provided in the catalog.

The accuracy of the PSF matching is crucial to our HST photometry. To test it, we extract stars from the CANDELS deep region (to ensure a relatively high $\mathrm{S} / \mathrm{N}$ ) from all PSF-matched HST images and compare their light profiles and curves of growth in Figure 6. The curves of growth of all HST bands, normalized by that of the F160W band, quickly converge to unity after a few pixels. If the radius of the isophotal aperture of a source is larger than 2 pixels $\left(0{ }^{\prime} .12\right)$, the relative error of isophotal fluxes in all HST bands is less than 5\%. Overall, our PSF matching does not induce a significant systematic offset for the bulk of our sources. Only $2.5 \%$ of sources in our sample have isophotal radii less than 2 pixels. For them, the relative photometric systematic offsets or uncertainties induced by PSF matching could be larger than $5 \%$. For these sources, we enforce a minimum aperture with radius of 2.08 pixels $\left(0{ }^{\prime \prime} 125\right)$. Fluxes within the minimum aperture (SExtractor parameter FLUX_APER) instead of FLUX_ISO are used for these sources in all $H S T$ bands. The aperture fluxes and uncertainties are then scaled up by a new aperture correction factor, apcorr $\equiv$ FLUX_AUTO/FLUX_APER of the F160W band, to convert into the total fluxes and uncertainties.

\subsection{HST Noise Properties}

The uncertainties of our HST photometry are essentially the SExtractor FLUXERR_ISO parameter scaled by an aperture correction factor. Recent works (e.g., Wuyts et al. 2008; Coe et al. 2013) have suggested that this parameter may underestimate the true photometric uncertainties by a significant factor (up to 2-3), primarily due to the pixel-to-pixel correlations that are induced by the step of drizzle (e.g., Casertano et al. 2000) in the image reduction/combination process.

However, in our case we provide SExtractor an rms map for each filter that has already been corrected for the correlations. Our pipeline that generates the drizzled HST images also produces a weight map whose value is nominally the inverse variance of the expected background noise, predicted using a noise model for the instrument that takes into account the background level, the exposure time, and the expected instrumental noise. In principle, this weight map should correctly describe the actual image noise. However, the noise in the drizzled science images is suppressed by the pixel-to-pixel correlations. We measure the background noise and the factor by which it is suppressed using an IRAF script "acall," originally developed for GOODS (M. Dickinson 2013, private communication). The script runs on a relatively empty region of the image with relatively uniform exposure time. After masking our objects in the image and removing any low-level background variations by median filtering on large angular scales, the script computes the autocorrelation function of the unmasked pixels. The twodimensional autocorrelation image typically has a strong peak and falls off sharply on a scale of a few drizzled pixels, since the data reduction (mainly drizzling) does not introduce correlations on scales much larger than 1 or 2 original detector pixels. The script measures the peak value of the autocorrelation image and the total power integrated out to some small radius that can be set interactively. The square root of this ratio (peak over total) is the rms suppression factor $\left(C_{s} \leqslant 1\right)$ due to the pixel-to-pixel correlations. The measured inverse variance, corrected for the 

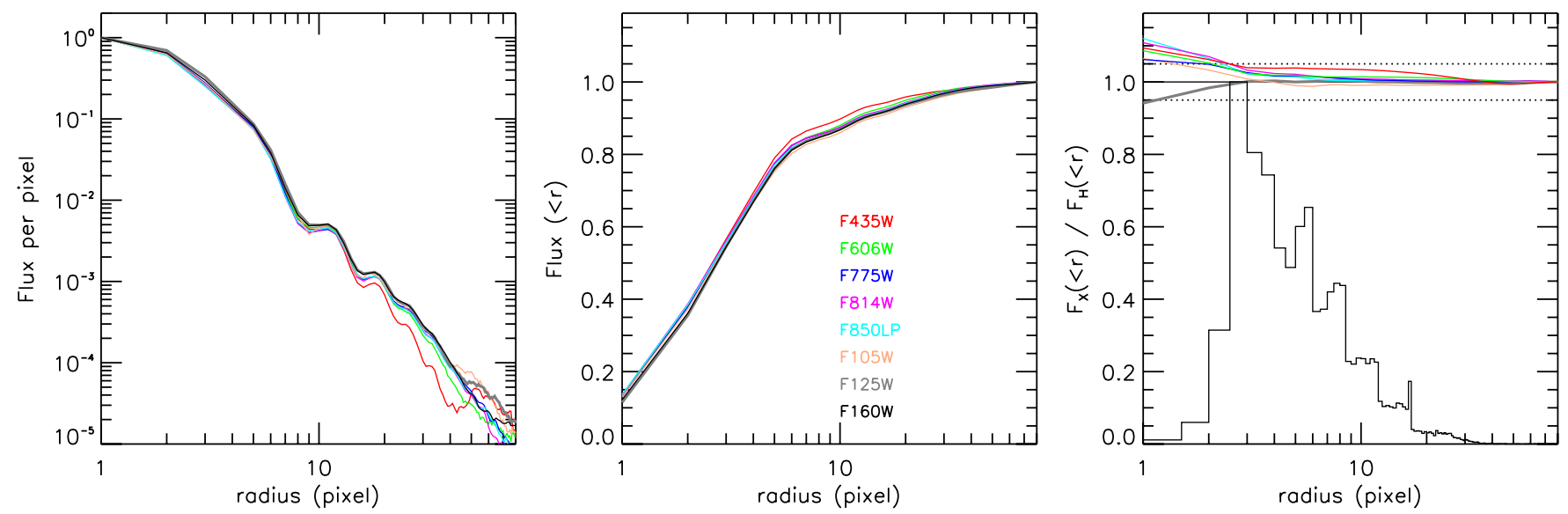

Figure 6. Accuracy of PSF matching between other HST bands and F160W. Left: the light profile of matched PSFs for each band. Middle: the curve of growth of each matched PSF. Right: the curve of growth of each matched PSF normalized by the curve of growth of the F160W PSF. In this panel, curves with values greater than unity are undersmoothed, and vice versa. All curves are color coded as labels in the middle panel show. Dotted lines in the right panel show the 5\% relative error. The solid histogram in the right panel shows the distribution of isophotal radii of all objects in our catalog.

(A color version of this figure is available in the online journal.)

correlations, is therefore $\left(C_{s} / \mathrm{rms}\right)^{2}$. This is compared to the value predicted from the weight map produced by the drizzle pipeline, and if necessary the weight map is re-scaled accordingly. The final rms map used for photometry is then computed from the inverse square root of the re-scaled weight map.

We carry out a test, equivalent to the "empty aperture" method of measuring the variance within empty apertures of various sizes placed throughout the images (e.g., Wuyts et al. 2008; Whitaker et al. 2011; Coe et al. 2013), to examine the noise properties of our $H S T$ images. First, we mask out all detected sources in the HST images. Then, for each band, we block sum both the drizzled science image and the variance map (square of the rms map) with various block sizes ( $r b$, in unit of pixels). Each block can be treated as an "empty box." We then calculate the ratio of the background rms of the summed science image and the median of the summed rms map (square root of the summed variance map). The ratio should be equal to unity if the pixel-to-pixel correlations are corrected.

Figure 7 shows the ratio as a function of $r b$ in the wide, deep, and HUDF regions of the F160W image. The ratio is close to 1 at $5<r b<13$, demonstrating that our rms map represents the background noise correctly on the scales relevant for most of our sources in the image, whose typical sizes are $\sim 7$ pixels. At $r b>13$, the background $\mathrm{rms}$ is larger than the median of the rms map, mainly due to the unmasked wings of detected objects and undetected sources, which contaminate most blocked pixels when $r b$ is large. It could also be due to any larger scale fluctuations not removed by the flat-fielding and sky subtraction steps of the data processing. This effect is strongest in the HUDF region because it has the highest source number density and detects more extended wings of objects than the other two regions.

The effect of correlation noise becomes more severe at smaller scale. At $r b=1$, the background rms is lower than the median of the rms map by a factor of $2 \sim 3$, implying that the unblocked pixel-to-pixel rms would significantly underestimate photometric uncertainties due to the correlation. This result is consistent with the test of Wuyts et al. (2008, see their Figure 3(a)). However, our photometric uncertainties are not measured from a linear scaling of the pixel-to-pixel rms of the drizzled science image. Instead, they are measured from the corrected rms map, which is made from the re-scaled

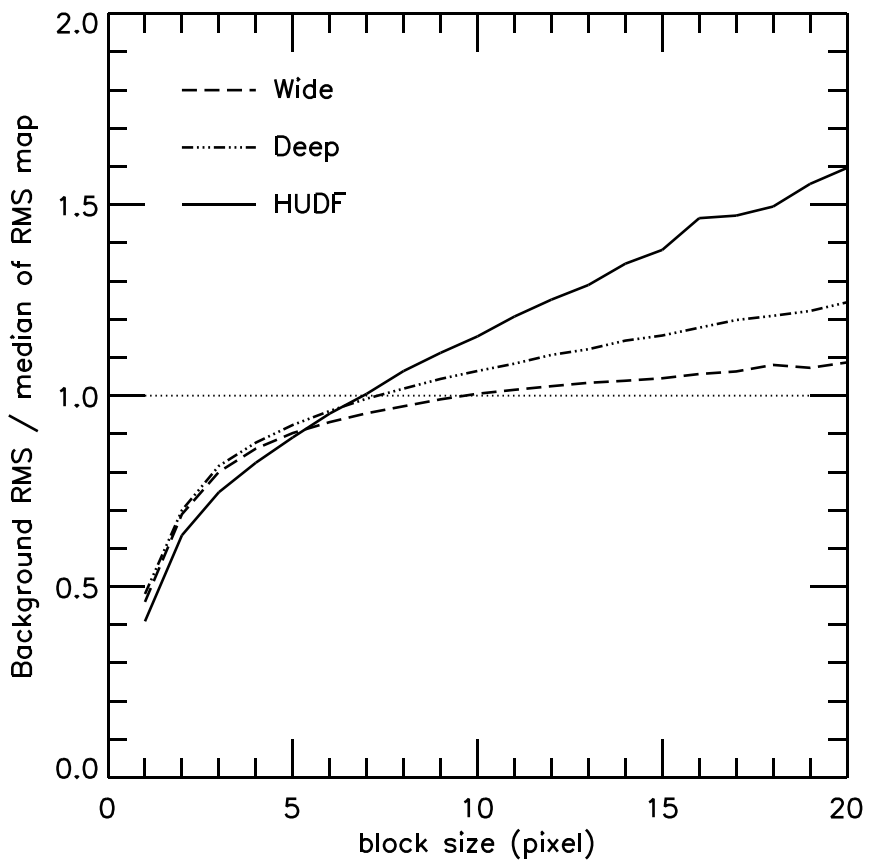

Figure 7. Ratio of the background rms of block summed F160W image and the median of the summed rms map (square root of the block summed variance maps) as a function of the block size.

weight map to represent the uncorrelated background fluctuation of our images. Therefore, we conclude that our photometric uncertainties in F160W are not underestimated by the pixelto-pixel correlations. The same test on other HST bands shows similar results.

Last, it is important to note that the HST photometric uncertainties in our catalog are most appropriate when the fluxes are used to compute colors in combination with other bands in this catalog. The HST S/Ns in our catalog are actually measured within the isophotal area and hence are higher than the total S/Ns measured from a larger aperture (e.g., Kron radius). If the $\mathrm{S} / \mathrm{Ns}$ in our catalog are used instead as an estimate of the total S/Ns in these bands, the photometric uncertainties should be inflated, roughly by the ratio of the F160W S/N in the AUTO aperture to the $\mathrm{S} / \mathrm{N}$ in the ISO aperture. 


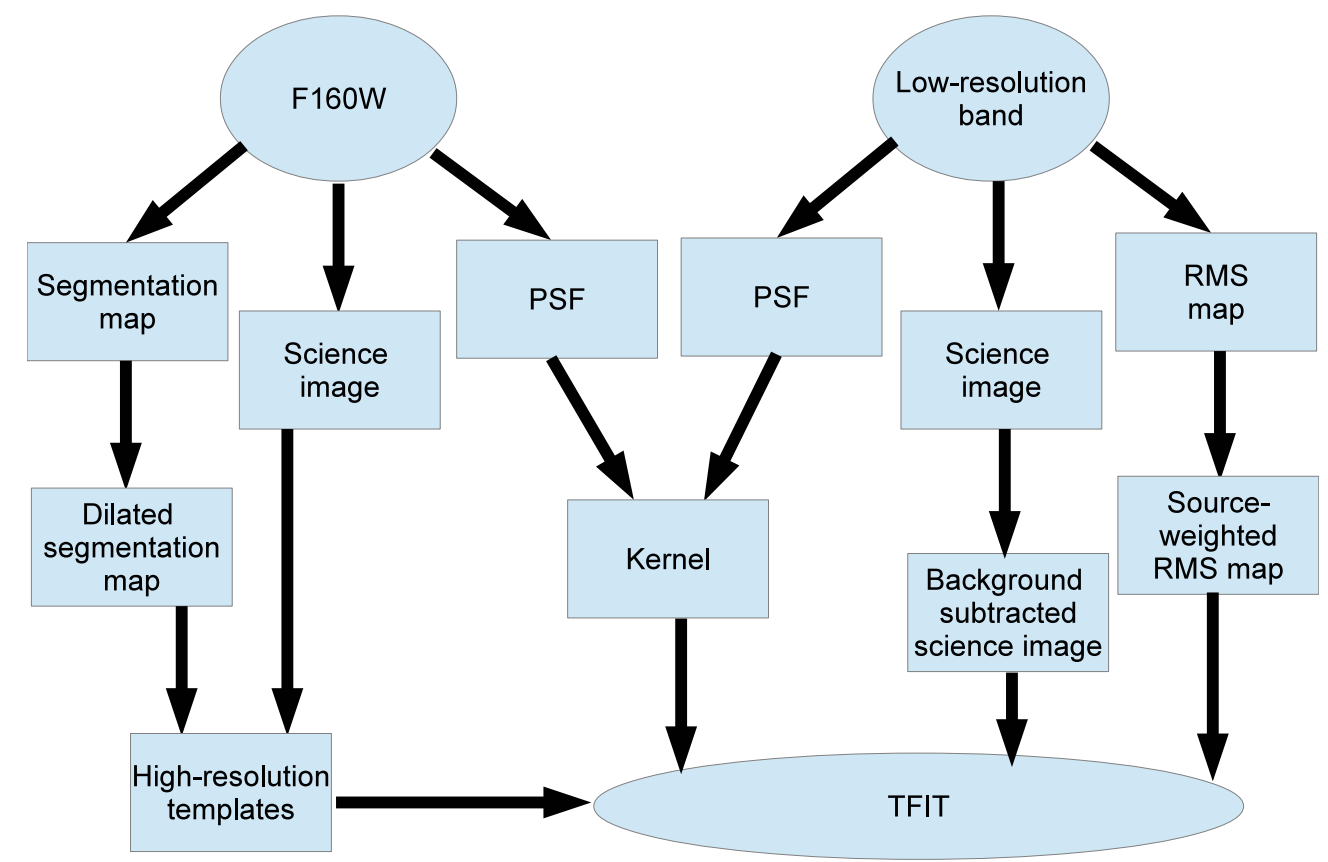

Figure 8. Flow chart of pre-processing of images prior to feeding them into TFIT for measuring photometry for low-resolution bands.

(A color version of this figure is available in the online journal.)

\section{PHOTOMETRY OF LOW-RESOLUTION IMAGES}

In a multi-wavelength survey, it has long been a challenge to measure reliable and uniform photometry for bands whose spatial resolutions differ dramatically. In GOODS-S, the FWHM of the PSFs varies from $\sim 0^{\prime} .17$ for $H S T$ images to $\sim 2^{\prime \prime} .0$ for IRAC $8.0 \mu \mathrm{m}$, a factor of $\sim 12$. Close pairs identified in a highresolution image could be blended into single sources in a lowresolution image if the separation between the companions is less than 1 FWHM of the low-resolution image. In order to measure the flux of each member of such pairs in the lowresolution image, a few methods have been used in the literature, e.g., the template fitting (Grazian et al. 2006; Laidler et al. 2007), the "clean" process migrated from radio astronomy (Wang et al. 2010), etc.

In our paper, we use a software package developed by the GOODS team, TFIT, to carry out the template-fitting method to measure low-resolution photometry. Details of TFIT are given by Laidler et al. (2007), while thorough tests on its robustness and uncertainties through multi-band simulations are given by Lee et al. (2012). For each object, TFIT uses the spatial position and morphology of the object in a high-resolution image to construct a template. This template is smoothed to match the resolution of the low-resolution image and fit to the lowresolution image of the object. During the fitting, flux is left as a free parameter. The best-fit flux is taken to be the flux of the object in the low-resolution image, and the variance of the fitted flux is the uncertainty in the flux. These procedures can be simultaneously done for several objects that are close enough to each other in the sky so that the blending effect of these objects on the flux measurement would be minimized. Experiments on both simulated and real images show that TFIT is able to measure accurate photometry of objects to the limiting sensitivity of the image.

There are several steps for pre-processing both highresolution and low-resolution images prior to feeding them to TFIT. Figure 8 presents a flow chart of these steps. Galametz et al. (2013) give a detailed description of each step. Here we only summarize some key steps.

1. High-resolution templates. For each source detected in our F160W mosaic, we cut out a postage stamp for it as the high-resolution template. The size of the postage stamp is dependent on the segmentation area generated by SExtractor. This segmentation area of a source, however, only contains pixels above the isophotal detection threshold and could result in an artificial truncation of the light profile of the object. Galametz et al. (2013) determined an empirical relation to extend the segmentation area of an F160W source to a proper size to include the outer wings of an object in the template. This process, called "dilation," was thoroughly tested and optimized by Galametz et al. (2013).

2. Convolution kernel. TFIT requires a convolution kernel to smooth the high-resolution templates to low resolution. We use the IRAF/PSFMATCH package to compute the kernel for ground-based bands (CTIO $U$, VIMOS $U$, and ISAAC $K s$ ). Our F160W PSF is a hybrid of the empirical PSF from stacking stars and the simulated PSF from TinyTim (van der Wel et al. 2012). Our ground-based low-resolution PSFs and IRAC 3.6 and $4.5 \mu \mathrm{m}$ PSFs are generated by stacking stars, and IRAC 5.8 and $8.0 \mu \mathrm{m}$ PSFs are model PSFs convolved with an empirical kernel to slightly broaden them. For IRAC channels, with FWHMs more than 10 times broader than that of F160W, we simply use the IRAC PSFs as kernels to smooth the F160W templates.

3. Low-resolution images. In principle, TFIT can fit both background and objects simultaneously. However, we choose to subtract the background of low-resolution images before running TFIT because fitting background would introduce strong degeneracy and large uncertainty to the photometry of faint sources. Galametz et al. (2013) give details of our background subtraction algorithm. We also run the "empty block" test described in Section 3.3 to examine the noise properties of the low-resolution images. In order to 


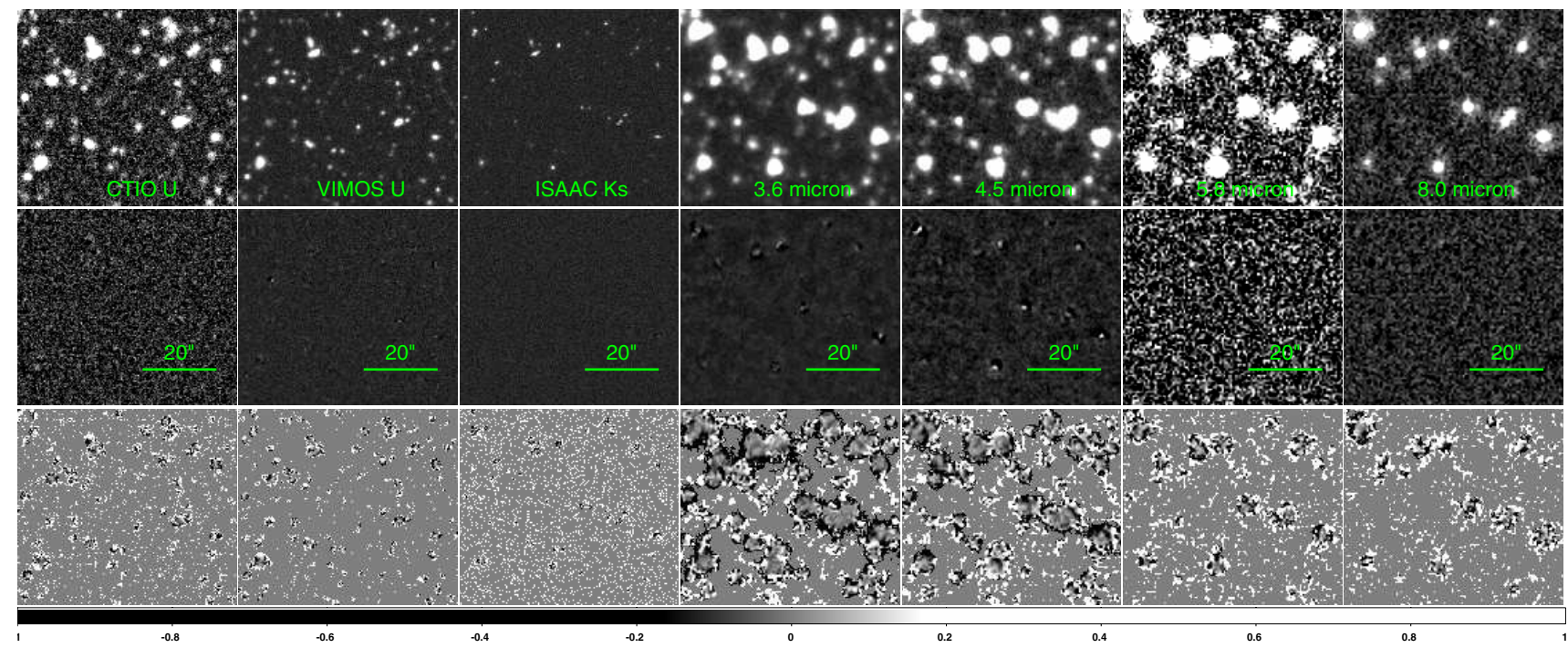

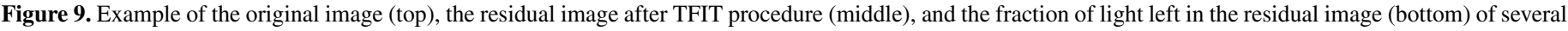

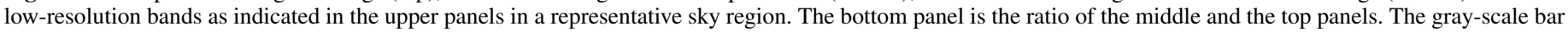
shows the contrast of the bottom panel.

(A color version of this figure is available in the online journal.)

correct the effect of the pixel-to-pixel correlations induced by the image reduction, we re-scale the rms map of each band so that the ratio of the background rms of the block summed image and the median of the square root of the block summed variance map is equal to unity at block sizes that are close to the typical sizes of sources in the image.

4. Special treatment for individual bands. Several lowresolution bands need special treatment. For both the CTIO/MOSAIC and VLT/VIMOS $U$ bands, if a source is covered by our ACS F435W image, we use the ACS F435W instead of the WFC3 F160W image as the highresolution template to minimize the effect of morphological change from the UV to NIR wavelengths. To keep the same detection sources, we still use the dilated F160W segmentation map for creating high-resolution postage stamps. About 2300 sources ( $\sim 7 \%$ of our catalog) are not covered by the ACS F435W image. For them, the F160W image is used as the template for both $U$ bands. For VLT/ISAAC $K s$ band, we run TFIT on each of its tiles instead of on the co-added mosaic because the tile-by-tile variation of PSF is non-negligible. We also run TFIT for each epoch of IRAC 5.8 and $8.0 \mu \mathrm{m}$ due to the PSF variation. For sources that are fitted more than once in the ISAAC and IRAC bands, we calculate a weight-averaged flux from each fitting using the squared fitting uncertainty as the inverse weight.

To correct the geometric distortion and/or misregistration between the high- and low-resolution images, we run TFIT in two passes. In the first pass, TFIT calculates a cross-correlation between the model image and the low-resolution image to determine the optimal shifted transfer kernels for each zone in the image. The second pass then uses these shifted kernels to smooth the high-resolution templates to the low resolution. The two-pass scheme effectively reduces the misalignment between templates and low-resolution images.

An example of the original images and residual maps of some TFIT bands is shown in Figure 9. A simple visual comparison between the original and the residual images suggests that TFIT does an excellent job of extracting light for the majority of sources in the low-resolution bands. The overall residual is quite close to zero in the maps of $U, K s$, and IRAC 5.8 and $8.0 \mu \mathrm{m}$. Some (especially bright) objects have obvious residuals left in the map of IRAC 3.6 and $4.5 \mu \mathrm{m}$. To quantify the residual, we calculate the fraction of light left in the residual maps (bottom panels). For most pixels in the sources with the worst residuals, the residual light is around $\pm 10 \%$ of the original signal. The average residual over the area of these sources, taking into account both positive and negative pixels, is actually quite close to zero, suggesting that the photometry of bright sources in IRAC 3.6 and $4.5 \mu \mathrm{m}$ is not significantly mismeasured, even though their residual maps look worse than others. The residual maps only provide a rough estimate of the quality of our photometry. A quantitative analysis of the quality of the catalog will be presented in Section 5.

After measuring the low-resolution photometry with TFIT, we merge the photometry of all the available bands, both low resolution and $H S T$, into the final catalog. This step is straightforward because each source keeps its ID and coordinates as determined in the F160W detection through the whole procedure of measuring its multi-band photometry. For each source, we also include a weight in each band. For HST and Spitzer bands, the weight is the mean exposure time within 6 pixels from the center of the object. For other bands, it is the average weight within 6 pixels from the center. The column descriptions of the catalog are given in Table 3.

\section{QUALITY CHECKS}

We test the quality of our catalog by (1) comparing colors of stars in our catalog and that in stellar libraries, (2) comparing our catalog with other published catalogs in GOODS-S, (3) measuring zero-point offsets through the best-fit templates of the SED of spectroscopically observed galaxies, and (4) evaluating the accuracy of photometric redshifts (photo- $z$ 's) measured with our catalog.

\subsection{Colors of Stars}

We first check the quality of our HST photometry by comparing the ACS-WFC3 colors of stars in our catalog with that of stars in stellar libraries. Because GOODS-S is located at high 
Table 3

Column Description of the CANDELS GOODS-S Catalog

\begin{tabular}{|c|c|c|}
\hline Column No. & Column Title & Description \\
\hline 1 & ID & Object identifier, beginning from 1 \\
\hline 2 & IAU Name & \\
\hline 3,4 & R.A., Decl. & Right ascension and declination (J2000.0; decimal degrees) \\
\hline 5 & F160W Limiting Magnitude & Limiting magnitude at the position of the source in the F160W image $\mathrm{e}^{\mathrm{a}}$ \\
\hline 6 & FLAGS & SExtractor F160W flag used to designate suspicious sources that fall in contaminated regions ${ }^{\mathrm{b}}$ \\
\hline 7 & CLASS_STAR & SExtractor CLASS_STAR parameter in the F160W band \\
\hline $8-58$ & Flux, Flux_Err, Weight & $\begin{array}{l}\text { Triplet of flux, flux uncertainty, and weight in each filter. Sources that are not observed have } \\
(-99.00,-99.00,0) \text {. } \\
\text { In } H S T \text { bands, the weight is the exposure time of the source, while in other bands, it is a relative } \\
\text { weight. } \\
\text { Filters are included in order: CTIO_U, VIMOS_U, F435W, F606W, F775W, F814W, F850LP, } \\
\text { F098M, F105W, F125W, F160W, ISAAC_Ks, HAWK-I_Ks, } 3.6 \mu \mathrm{m}, 4.5 \mu \mathrm{m}, 5.8 \mu \mathrm{m} \text {, and } 8.0 \mu \mathrm{m}\end{array}$ \\
\hline 59,60 & FLUX_ISO, FLUXERR_ISO & F160 isophotal flux and flux error ${ }^{c}$ \\
\hline 61,62 & FLUX_AUTO, FLUXERR_AUTO & F160 AUTO flux and flux error \\
\hline 63 & FWHM_IMAGE & FWHM of the F160W image of object, in unit of pixel (1 pixel $=0.06$ ) \\
\hline 64,65 & A_IMAGE, B_IMAGE & F160W profile rms along major and minor axes (pixel) \\
\hline 66 & KRON_RADIUS & F160W band Kron radius from SExtractor (in unit of A_IMAGE or B_IMAGE) \\
\hline $67,68,69$ & FLUX_RADIUS & F160W band FLUX_RADIUS with the fraction of light of $0.2,0.5$, and 0.8 from SExtractor (pixel) \\
\hline 70 & THETA_IMAGE & F160W position angle (degree) \\
\hline 71 & Apcorr & Ratio of SExtractor FLUX_AUTO and FLUX_ISO in the F160W band \\
\hline 72 & HOT_FLAG & $\begin{array}{l}\text { A flag to designate if the source enters the catalog as detected in the cold mode }(=0) \text { or in the hot } \\
\text { mode }(=1)\end{array}$ \\
\hline 73 & ISOAREAF_IMAGE & SExtractor F160W Isophotal area (filtered) above detection threshold, pixel ${ }^{2}$ \\
\hline
\end{tabular}

Notes.

a The limiting magnitude here is derived as $m_{\lim }=-2.5 \times \log _{10}\left(\sqrt{A<\sigma^{2}>}\right)+z p$, where $\left\langle\sigma^{2}\right\rangle$ is the average of the squared rms in the SExtractor F160W segmentation map of each source, $A$ is the area of $1 \operatorname{arcsec}^{2}$, and $z p$ is the zero point of F160W.

b A non-contaminated source has a flag of " 0 ." Sources detected on star spikes, halos, and the bright stars that produce those spikes and halos have a flag of "1." Sources detected by SExtractor at the image edges or on the few artifacts of the F160W image are assigned a flag of "2." Sources with both the flag of "1" and "2" have a flag of " 3 ."

${ }^{\mathrm{c}}$ For sources whose isophotal radius smaller than 2.08 pixels, these parameters are replaced by FLUX_APER and FLUXERR_APER measured within a radius of 0'. 125 (2.08 pixels). See Section 3.2 for details.

Galactic latitude, a large fraction of its stars may be metal-poor halo stars. The ACS-WFC3 colors, namely, the optical-NIR colors, of stars strongly depend on the metallicity of the stars because the optical fluxes are heavily absorbed by metals while the NIR fluxes are much less affected. Therefore, the ACS-WFC3 colors of stars in GOODS-S may be systematically different from the colors of stars in some commonly used stellar libraries, which are mainly calibrated with bright disk stars with richer metallicity. We use the model of stellar population synthesis of our Milky Way of Robin et al. $(2003)^{27}$ to estimate the metallicity distribution of stars in GOODS-S and find a median value of $[\mathrm{Fe} / \mathrm{H}] \sim-0.5$. We then choose the set of models with metallicity $[\mathrm{M} / \mathrm{H}]=-0.5$ from the synthesis stellar library of Lejeune et al. (1997) as our standard reference of stellar colors.

Figure 10 shows the locations of stars in our catalog and those in the Lejuene library in ACS-WFC3 color-color diagrams. Stars are selected with the SExtractor parameter STAR_CLASS $>0.98$ in the F160W band. For each color, we only use stars with $\mathrm{S} / \mathrm{N}>3$ in the two bands used to calculate the color. If the stellar photometry of one WFC 3 band (denoted by $\mathrm{X})$ is incorrectly measured, the (F775W-X) color of observed stars would deviate vertically from that of library stars in both $(\mathrm{F} 775 \mathrm{~W}-\mathrm{X})$ versus $(\mathrm{F} 435 \mathrm{~W}-\mathrm{F} 850 \mathrm{LP})$ and $(\mathrm{F} 775 \mathrm{~W}-\mathrm{X})$ versus (F435W-F775W) diagrams by a similar amount. In another situation, if the photometry of one of the ACS Biz bands is mismeasured, the observed stellar color would deviate horizontally by a similar amount from that of library stars in all diagrams of (F775W-X) versus ACS color, where the ACS color involves

\footnotetext{
27 http://model.obs-besancon.fr
}

the problematic band. There is excellent agreement between our observed stars and library stars for all colors in the plot, suggesting that our stellar ACS-WFC3 colors are accurate.

\subsection{Comparison with Other Catalogs}

We also compare our photometry with other published multiwavelength catalogs in GOODS-S. The comparison helps in two ways. First, it provides an assessment of the photometry of extended sources. Second, although we find no systematic offset in our stellar ACS-WFC3 colors, we still need an absolute measurement of the fluxes of either ACS or WFC3 bands.

Two multi-wavelength catalogs in the literature cover (almost) the entire GOODS-S field as well as include most of our bands: GOODS-MUSIC (GM; Grazian et al. 2006; Santini et al. 2009) and FIREWORKS (FW; Wuyts et al. 2008). GM includes ACS F435W, F606W, F775W, and F850LP images, VLT JH Ks data, Spitzer/IRAC (3.6, 4.5, 5.8, and $8.0 \mu \mathrm{m})$ and MIPS $(24 \mu \mathrm{m})$ data, and publicly available $U$-band data from the ESO $2.2 \mathrm{~m}$ telescope and VLT/VIMOS. A software package, ConvPhot, which operates on the same principle as TFIT, but is a completely independent implementation, has been developed for measuring PSF-matching photometry for space- and ground-based images of different resolutions and depths. Different from our detection scheme, GM detects objects mainly in F850LP and is supplemented by $K s$ and $4.5 \mu \mathrm{m}$ band detected sources.

FW is a $K s$-selected catalog for the CDF-S, containing photometry in the ACS F435W, F606W, F775W, and F850LP, ground-based $U, B, V, R$, and $I$, VLT $J, H$, and $K s$, 


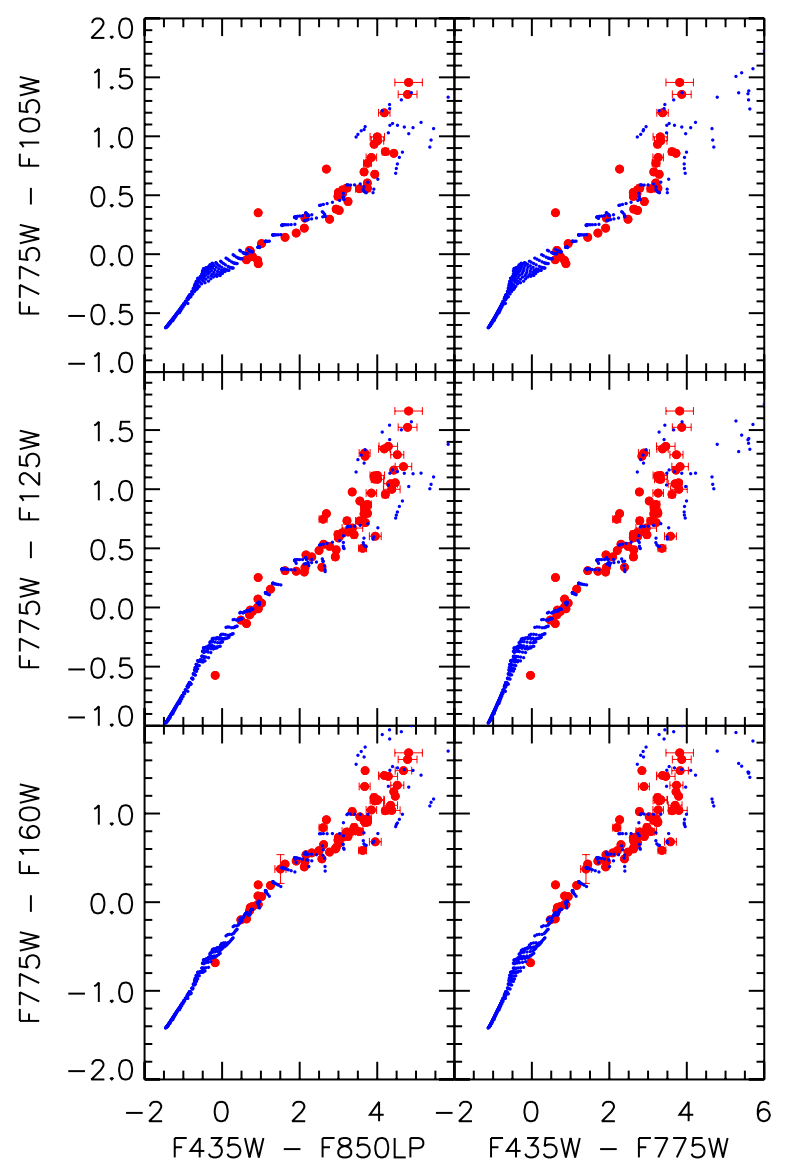

Figure 10. Comparison between colors of stars in our catalog (red points with error bars) and that of stars in the synthetic library of Lejeune et al. (1997) (blue). Colors are in AB magnitudes as indicated.

(A color version of this figure is available in the online journal.)

IRAC 3.6, 4.5, 5.8, and $8.0 \mu \mathrm{m}$, and MIPS $24 \mu \mathrm{m}$ bands. Photometry in ACS and ISAAC bands is measured using SExtractor in dual-image mode with the $K s$-band mosaic as the detection image. Color and aperture photometry are measured with the same apertures in each band. SExtractor MAG_AUTO is used to derive the total flux of the $K s$-detected objects. An aperture correction is applied to compute the total integrated flux based on the curve of growth of the PSF of the $K s$ band. The totalto-aperture correction factor of the $K s$ band is then applied to the other ACS and ISAAC bands. Photometry in IRAC bands is measured with the method of Labbé et al. (2006). Similar to TFIT, it fits the light profile of the higher resolution image to that of lower resolution images and leaves flux as a free parameter. For a single source, however, the method does not use the best-fit flux as the total flux of the source. Instead, it subtracts its neighbors based on the best-fit models and then estimates the flux of the source of interest via aperture photometry.

In order to compare our photometry with that in the two catalogs, we match our CANDELS GOODS-S catalog (CGS) to the other two through source coordinates with a maximum matching radius of 0.3 and 0 .'5 for GM and $\mathrm{FW}$, respectively. We only use cleanly detected sources (i.e., not saturated, not truncated, not having bad pixels, etc.) in our catalog with the F160W SExtractor parameter FLAG $=0$. The comparisons are shown in Figure 11. For each band, we only consider objects with $\mathrm{S} / \mathrm{N}>3$ in our and the other catalogs.

On average, there is a good agreement with nearly zero systematic offset between CGS and the other two catalogs over the magnitude range to $\sim 24 \mathrm{AB}$ mag in most bands (except the IRAC 5.8 and $8.0 \mu \mathrm{m})$. The offset reaches $\sim 0.05 \mathrm{mag}$ in the worst bands. At the faint end ( $>24 \mathrm{AB}$ mag), deviations are evident between CGS and the other two.

At the very faint end (the shaded areas in Figure 11), the Eddington bias makes the comparison unreliable. The flux uncertainties of objects in a shallower catalog (GM and FW) are larger so that objects in it are more likely to be scattered to brighter or fainter magnitudes than in a deeper catalog. Sources close to the $\mathrm{S} / \mathrm{N}=3$ limit in CGS should be included in the comparison. In fact, however, if the fluxes of their counterparts in GM or FW are scattered toward fainter fluxes, these sources are excluded because their $\mathrm{S} / \mathrm{N}$ in $\mathrm{GM}$ or $\mathrm{FW}$ is now $<3$. Therefore, at the $\mathrm{S} / \mathrm{N}$ limit, only those CGS sources whose GM and FW fluxes are scattered toward brighter fluxes are included in the comparison. This biases the comparison. In order to determine the magnitude range subjected to Eddington bias in each band, we fit a power law to the differential number count density of $\mathrm{GM}$ and $\mathrm{FW}$ without any $\mathrm{S} / \mathrm{N}$ cut. After the $\mathrm{S} / \mathrm{N}>3$ cut is applied, magnitude ranges where the new differential number count density is less than $50 \%$ of the best-fit power law are now very incomplete in GM and FW and hence induce Eddington bias. The comparisons in these magnitude ranges are not discussed here because they are unreliable.

Finally, all comparisons in this section only show the consistency/inconsistency between our and the other two catalogs. They cannot tell us whether one catalog is more accurate than the others.

\subsubsection{UV to Optical Photometry}

There is an almost constant offset of $\sim-0.05$ mag between CGS and GM in the VIMOS $U$ band to $\sim 27 \mathrm{AB}$ mag, where CGS measures brighter magnitudes. This deviation could be caused by the different high-resolution templates and kernels used in the template profile fitting method of both catalogs. In GM, ACS F850LP images are used as the templates to fit the $U$-band image, while in CGS, ACS F435W images are used to minimize the wavelength-dependent morphological changes. To provide an independent check, we compare our CGS photometry with the SExtractor MAG_AUTO photometry of Nonino et al. (2009). We find good agreement with almost zero offset over the magnitude range to $\sim 27 \mathrm{AB}$ mag.

In the ACS bands, the agreement between our and the other two catalogs is quite good. The offset between CGS and GM is almost zero for objects with magnitude brighter than $24 \mathrm{AB}$ mag. The largest offset, seen in the ACS F606W band, is about $-0.02 \mathrm{mag}$. For objects fainter than $24 \mathrm{AB}$ mag, CGS magnitudes are brighter, and the deviation between the two catalogs increases toward fainter magnitude. The deviation may be due to different apertures used to measure the total fluxes in CGS and GM. The aperture size, represented by the KRON_RADIUS in SExtractor, is defined in the F160W band in CGS and then applied to other HST bands through our aperture correction factors, assuming no morphological changes between these bands. In GM, however, the KRON_RADIUS is defined in the ACS F850LP band. Due to the high sensitivity and broader PSF of WFC3, the KRON_RADIUS defined in the F160W band is typically larger than that defined in the F850LP band for each individual source. Therefore, the F160W KRON_RADIUS counts more light from the wings of each object and measures a brighter magnitude. In fact, we find an obvious correlation between the difference of magnitudes and the difference of KRON_RADIUS. Objects whose F160W 

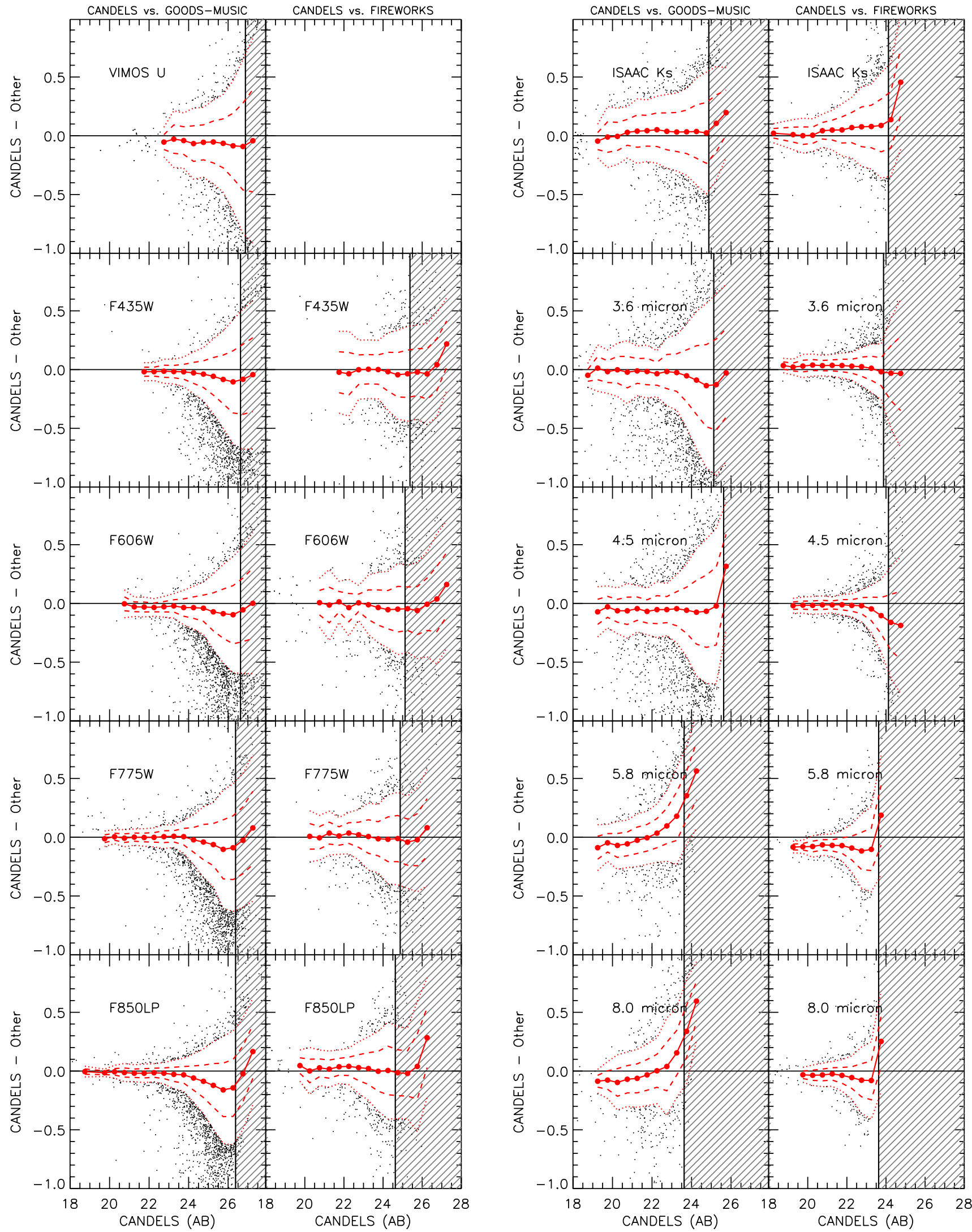

Figure 11. Comparison between our photometry ("CANDELS") and two published catalogs ("Other") in GOODS-S: GOODS-MUSIC (first and third columns) and FIREWORKS (second and fourth columns). For each band, we only use sources with $\mathrm{S} / \mathrm{N}>3$ in both our and the other catalog for comparison. In each panel, red points connected by solid line show the mean (after $3 \sigma$ clipping) of the magnitude difference (defined as CANDELS - Other) as a function of magnitude. Upper and lower red dash/dotted lines show $1 \sigma / 2 \sigma$ confidence level after $3 \sigma$ clipping of the magnitude difference. Black dots show objects whose magnitude difference is beyond $2 \sigma$ of the mean. The shaded area of each panel is subjected to the Eddington bias (see the text and discussion on Figure 13 for details) so the comparison there is biased.

(A color version of this figure is available in the online journal.) 


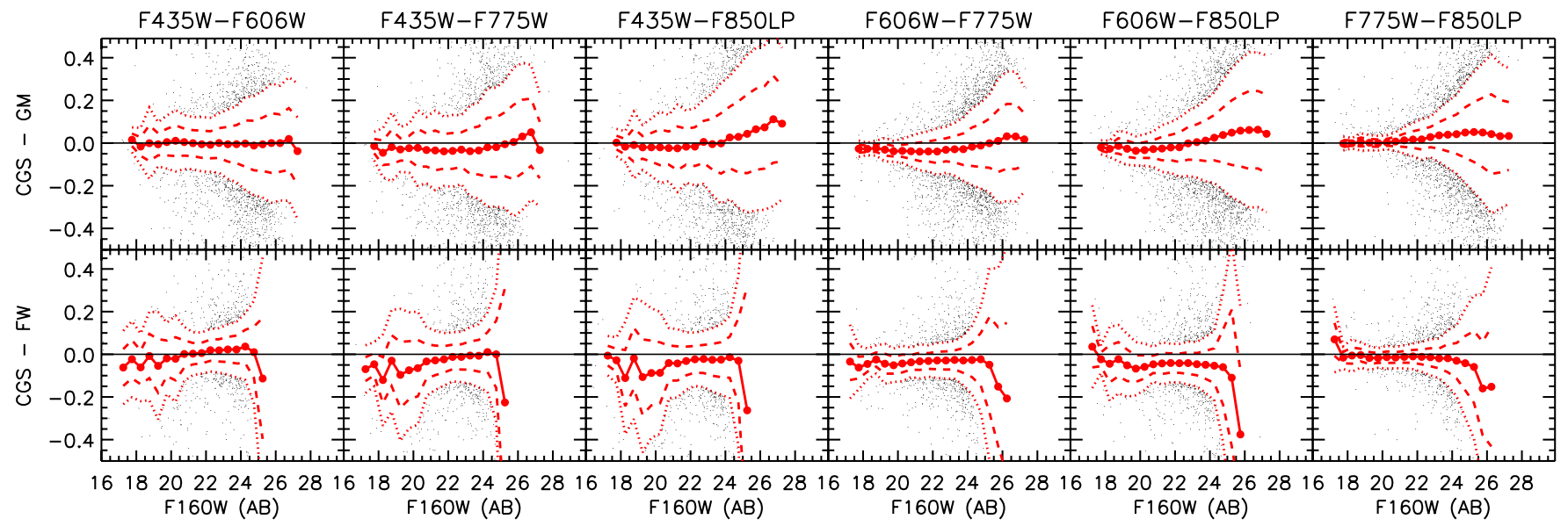

Figure 12. Differences of ACS colors measured by our catalog (CGS) and by GOODS-MUSIC (GM, upper panels) and FIREWORKS (FW, lower panels). In each panel, red points connected by a solid line show the mean (after $3 \sigma$ clipping) difference of one ACS color in CGS and the other catalog as a function of the F160W magnitude. Upper and lower red dash/dotted lines show $1 \sigma / 2 \sigma$ confidence level after $3 \sigma$ clipping of the magnitude difference. Black dots show objects whose magnitude difference is beyond $2 \sigma$ of the mean. We only use sources with $\mathrm{S} / \mathrm{N}<5$ in ACS and F160W bands in the comparison.

(A color version of this figure is available in the online journal.)

KRON_RADIUS is larger than the F850LP one are also brighter in our catalog, and vice versa. This correlation supports our speculation that KRON_RADIUS defined in different images is the reason for the deviation in the faint end between CGS and GM. More supportive evidence is from the excellent agreement between the ACS colors of CGS and GM (the upper panels of Figure 12). Colors from both catalogs have almost zero $(\sim 0.02$ mag in the worst case for sources brighter than $26 \mathrm{AB}$ mag in $\mathrm{F} 160 \mathrm{~W}$ ) offset over the magnitude range to F160W $\sim 26 \mathrm{AB}$ mag. This excellent agreement demonstrates that our PSF-matching process does not induce a systematic offset to our ACS photometry. Therefore, the offset in the faint end of ACS photometry between the two catalogs is likely caused by the different apertures used to measure fluxes.

The comparison between CGS and FW shows large scatter, possibly due to both small number statistics and different photometry measurements. FW has a faint limit cut on $24.3 \mathrm{AB}$ mag in its detection $K s$ band, which excludes the majority of sources fainter than $25 \mathrm{AB}$ mag in the ACS bands in our catalog. Therefore, the comparison fainter than $25 \mathrm{AB}$ mag has large uncertainty due to small number statistics. Also, FW was generated based on an early version of the GOODS-S ISAAC $K s$-band image, which covers $\sim 130 \operatorname{arcmin}^{2}$, only $75 \%$ of the whole GOODS-S region. The smaller sky coverage further reduces the number of matched sources in this comparison. For sources brighter than $26 \mathrm{AB}$, the comparison has larger scatter, $\sim 0.15-0.2$, than that in the CGS versus GM comparison. The large scatter is likely due to the treatment of isolated sources and blended sources in the $K s$ band in FW. For isolated sources, FW used MAG_AUTO as the total flux with KRON_RADIUS defined in $K s$ band. Because the KRON_RADIUS defined in the $K s$ band is typically larger than that defined in the HST bands, isolated sources tend to have brighter magnitudes in FW than in CGS. On the other hand, to reduce the influence of neighboring sources, FW used a reduction factor to shrink the isophotal area of blended sources and used the isophotal flux to derive the total flux. Therefore, the fluxes of blended sources are likely underestimated in FW. The combination of the above two introduces large scatter to our comparison, as the plot shows. But overall, the mean difference between FW and CGS is close to zero over the magnitude range to $\sim 25 \mathrm{AB}$ mag, where the Eddington bias begins to affect the comparison.

\subsubsection{IR Photometry}

In the ISAAC $K s$ band, CGS shows a magnitude-dependent deviation from both GM and FW. CGS becomes fainter than the other two at $\sim 20 \mathrm{AB}$ mag. The deviation increases as the flux of sources decreases. At $\sim 24 \mathrm{AB}$ mag, the deviation reaches $\sim 0.05 \mathrm{mag}$ in CGS versus GM and $\sim 0.1 \mathrm{mag}$ in CGS versus FW. Due to the lack of sources with $\mathrm{S} / \mathrm{N}>5$ at magnitude fainter than $24 \mathrm{AB}$ mag, the deviation in the very faint end cannot be investigated. The deviation could be due to oversubtraction of background in the ISAAC images during our preprocessing of images (Section 4). However, measuring global background from the images used by TFIT shows no significant negative background, suggesting that our background subtraction pipeline does a fairly good job. The deviation in CGS versus FW could also be due to the underdeblending of neighboring sources in SExtractor-type photometry. Contaminating light from poorly separated companions would boost the photometry of faint sources but have little effect on bright sources.

CGS shows good agreement with GM and FW over the magnitude range to $24 \mathrm{AB}$ mag in IRAC 3.6 and $4.5 \mu \mathrm{m}$. The deviation between CGS and GM is almost zero at $3.6 \mu \mathrm{m}$ and about $-0.04 \mathrm{mag}$ at $4.5 \mu \mathrm{m}$, while that between CGS and FW is about $0.03 \mathrm{mag}$ at $3.6 \mu \mathrm{m}$ and almost zero at $4.5 \mu \mathrm{m}$. The absolute uncertainty in the IRAC calibration is about $3 \%$. Therefore, we believe that within the calibration uncertainty, there is no major concern on our photometry in the magnitude range of 18-24 AB mag for the IRAC 3.6 and $4.5 \mu \mathrm{m}$.

The comparison in the IRAC 5.8 and $8.0 \mu \mathrm{m}$ can only be done for sources brighter than $23 \mathrm{AB}$ mag. The deviations between CGS and GM in both channels are quite similar and magnitude dependent. CGS is brighter than GM by $\sim 0.1 \mathrm{mag}$ at $19 \mathrm{AB}$ mag and becomes fainter than GM by $\sim 0.05 \mathrm{mag}$ at $23 \mathrm{AB}$ mag. In contrast, the deviations between CGS and FW are almost constant, with CGS being brighter by $\sim 0.07$ mag and $\sim 0.03 \mathrm{mag}$ in the 5.8 and $8.0 \mu \mathrm{m}$ bands, respectively.

Although all three catalogs use the profile template-fitting method to derive the photometry of IRAC bands, the implementation of this method in each catalog is quite different. The scatter in CGS versus FW is significantly smaller than that in CGS versus GM. We suspect that the difference in scatter originates from the choice of high-resolution templates. NIR images are used in both CGS (F160W) and FW (Ks band) as the templates 


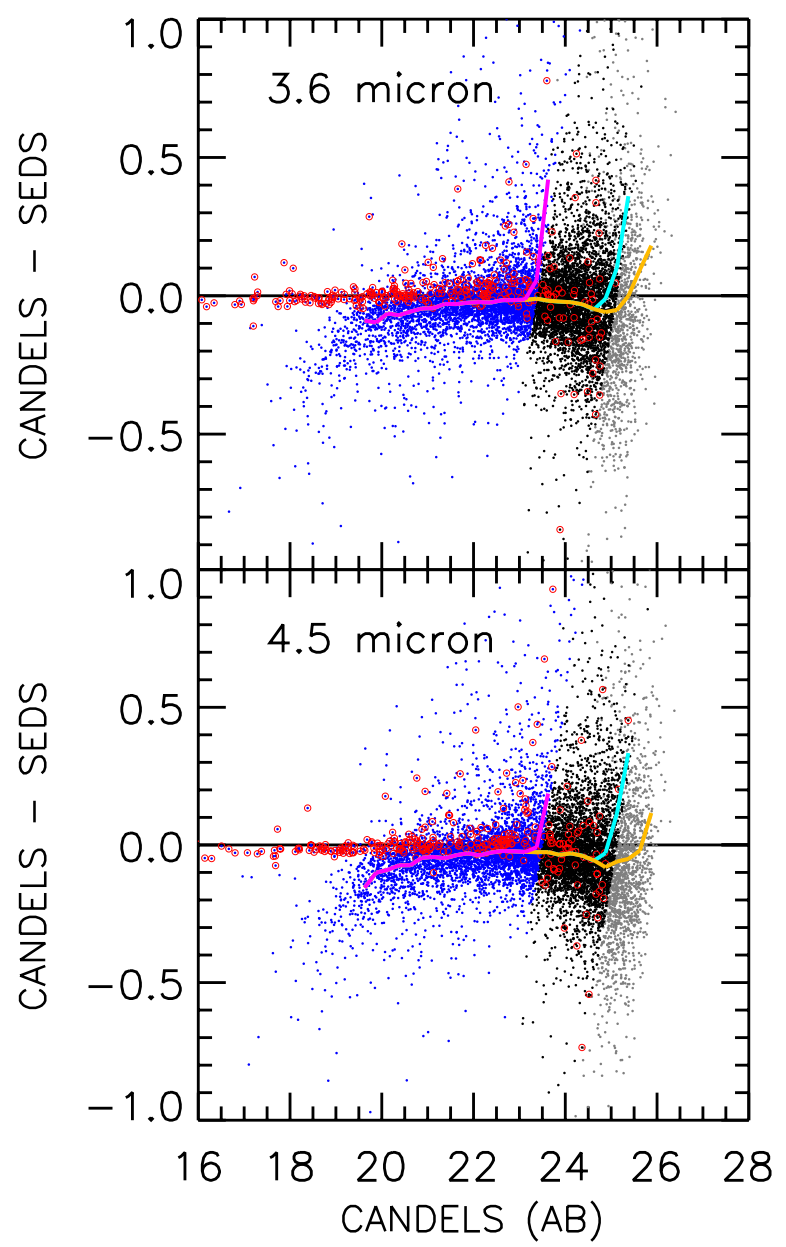

Figure 13. Comparison between CANDELS TFIT photometry and SEDS photometry for the IRAC 3.6 (upper) and $4.5 \mu \mathrm{m}$ (lower) bands. Points with different colors represent sources with various $\mathrm{S} / \mathrm{N}$ cuts on both catalogs: $\mathrm{S} / \mathrm{N}>8$ (blue), $\mathrm{S} / \mathrm{N}>5$ (black), and $\mathrm{S} / \mathrm{N}>3$ (gray). Red circles show point-like objects with $\mathrm{S} / \mathrm{N}>5$ and F160W SExtractor parameter STAR_CLASS $>0.8$. The average differences of the comparisons with various $\mathrm{S} / \mathrm{N}$ cuts are shown by colored curves: violet for $\mathrm{S} / \mathrm{N}>8$, cyan for $\mathrm{S} / \mathrm{N}>5$, and yellow for $\mathrm{S} / \mathrm{N}>3$. The large deviation at the faint end of each curve demonstrates the Eddington bias caused by the imposed $\mathrm{S} / \mathrm{N}$ cuts on both catalogs.

for fitting IRAC images. Although the resolution differs between the two sets of templates, the morphological change from them to IRAC bands would be quite small. On the other hand, GM uses the ACS $z$ band as templates to fit the profile of sources in IRAC bands. Since the templates and the IRAC images sample two distinct wavelength regimes, the morphological change could induce large difference in photometry, including systematic offset in the 5.8 and $8.0 \mu \mathrm{m}$ bands, where the wavelength difference between templates and sources becomes largest.

We also compare our TFIT photometry to the official SEDS catalog (Ashby et al. 2013) for IRAC 3.6 and $4.5 \mu \mathrm{m}$ bands. Ashby et al. used StarFinder to fit PSFs to sources and subtracted the best-fit PSFs from the original image. Then, for each source, they added its best-fit PSF back to the residual map while keeping other sources subtracted. They measured aperture photometry for the source with a variety of aperture sizes (here we choose the one with size of 2 ."4) and applied a correction factor to convert the aperture photometry to the total photometry based on the curve of growth of the IRAC PSFs. The comparison of CANDELS TFIT photometry and SEDS photometry is shown in Figure 13. For point-like sources (F160W SExtractor parameter STAR_CLASS $>0.8$ ) in both IRAC channels, the agreement between both catalogs is excellent over the magnitude range to $24.5 \mathrm{AB}$ mag with an offset of $\sim-0.04 \mathrm{mag}$. For bright ( $<20 \mathrm{AB}$ mag) extended sources, however, SEDS underestimates their fluxes. This is because the aperture correction used in SEDS is only valid for point sources. When using larger apertures (e.g., size of 6."0), SEDS photometry of bright extended sources agrees with our TFIT photometry much better with deviation $\sim 0.05 \mathrm{mag}$. Ashby et al. (2013) give a detailed description and analysis of SEDS photometry and its uncertainty.

Figure 12 also illustrates the Eddington bias in comparing two catalogs. Sources near the threshold will randomly have greater $\mathrm{S} / \mathrm{N}$ in one catalog than the other. Imposing an $\mathrm{S} / \mathrm{N}$ cutoff means that sources just above the cutoff will on average have catalog fluxes that are larger than the true values. If the uncertainties of the two catalogs differ, the amount of bias will differ. This effect can be seen in the figure as the sloping transition from blue $(\mathrm{S} / \mathrm{N}>8)$ to black $(\mathrm{S} / \mathrm{N}>5)$ and from black $(\mathrm{S} / \mathrm{N}>$ $5)$ to gray $(\mathrm{S} / \mathrm{N}>3)$ points. The deviation of $\sim 0.05 \mathrm{mag}$ at 23.5 AB mag for curves with $\mathrm{S} / \mathrm{N}>8$ is strongly biased and hence does not suggest any true difference between the two catalogs at this magnitude. In fact, good agreement between the two catalogs can be seen down to $25.5 \mathrm{AB}$ mag where the $\mathrm{S} / \mathrm{N}>$ 3 cut induces the Eddington bias.

Due to the Eddington bias, it is difficult to evaluate the accuracy of our IRAC photometry at the very faint end through comparisons with other catalogs. Instead, we use fake source simulations to test our photometry at the detection limit of the IRAC channels. We generate 1000 fake F160W sources and place them randomly into our F160W mosaic. We then use our TFIT pipeline to measure the IRAC photometry of these fake sources and real sources simultaneously. Because there are no counterparts to these fake sources in the IRAC images, on average their IRAC photometry should be zero. Indeed, the distributions of the ratio of IRAC photometry and its uncertainties in these fake sources in all IRAC channels have a mean of zero and a standard deviation of one. This result demonstrates that-assuming no PSF or registration mismatches - our catalog accurately measures IRAC photometry and its uncertainties down to the detection limits of IRAC images.

\subsection{Zero-point Offsets}

To further assess the overall accuracy of our photometry and its influence on deriving properties of galaxies, we measure the zero-point offset of each band by comparing the SEDs of spectroscopically observed galaxies to synthetic stellar population models. If a band suffers from significant systematic bias, its photometry will statistically deviate from the best-fit models.

The spectroscopic sample used in our study is from Dahlen et al. (2010). We match the sample to our F160W band coordinates with a maximum matching radius of 0.3 . We only use galaxies with good (flag $=1$ in Dahlen et al. 2010) spectroscopic redshifts (spec-z's) and exclude X-ray detected sources. The sample contains 1338 galaxies. The synthetic stellar population models used in this test are retrieved from the PEGASE v2.0 library (Fioc \& Rocca-Volmerange 1997). Details of SED fitting can be found in Guo et al. (2012). For each galaxy, we fix its redshift to its spec- $z$ during the fitting.

The template fitting can suffer from systematic errors if the models do not match real galaxy SEDs or if the absolute calibrations of the observations are systematically wrong at different wavelengths. In order to correct for such errors, we shift the observed and best-fit SEDs of each galaxy to 
Table 4

Zero-point Offset Derived from Best-fit SED Models

\begin{tabular}{lcc}
\hline \hline Band & $\begin{array}{c}\text { Zero-point Offset }^{\mathrm{a}} \\
\text { (Before Template Correction) }\end{array}$ & $\begin{array}{c}\text { Zero-point Offset } \\
\text { (After Template Correction) }\end{array}$ \\
\hline U_CTIO & $0.03 \pm 0.07$ & $0.03 \pm 0.07$ \\
U_VIMOS & $-0.01 \pm 0.06$ & $-0.01 \pm 0.06$ \\
F435W & $-0.03 \pm 0.08$ & $-0.03 \pm 0.08$ \\
F606W & $-0.01 \pm 0.05$ & $-0.01 \pm 0.05$ \\
F775W & $-0.01 \pm 0.05$ & $-0.01 \pm 0.04$ \\
F814W & $-0.01 \pm 0.05$ & $-0.01 \pm 0.05$ \\
F850LP & $-0.03 \pm 0.05$ & $-0.02 \pm 0.04$ \\
F098M & $0.02 \pm 0.05$ & $0.03 \pm 0.04$ \\
F105W & $0.01 \pm 0.04$ & $0.01 \pm 0.04$ \\
F125W & $0.01 \pm 0.05$ & $0.01 \pm 0.03$ \\
F160W & $0.02 \pm 0.05$ & $0.00 \pm 0.04$ \\
ISAAC $K s$ & $0.08 \pm 0.12$ & $0.06 \pm 0.12$ \\
HAWK-I $K s$ & $0.02 \pm 0.08$ & $0.01 \pm 0.07$ \\
IRAC 3.6 $\mu \mathrm{m}$ & $-0.09 \pm 0.10$ & $-0.07 \pm 0.10$ \\
IRAC $4.5 \mu \mathrm{m}$ & $-0.08 \pm 0.12$ & $-0.03 \pm 0.10$ \\
IRAC 5.8 $\mu \mathrm{m}$ & $-0.11 \pm 0.23$ & $-0.04 \pm 0.21$ \\
IRAC $8.0 \mu \mathrm{m}$ & $-0.31 \pm 0.49$ & $-0.20 \pm 0.47$ \\
\hline
\end{tabular}

Note. ${ }^{a}$ Offsets are defined as observed magnitude minus model magnitude. A positive offset means our photometry is fainter than models and vice versa.

rest-frame wavelength and calculate the offset between them as a function of rest-frame wavelength. The average offset of the spec- $z$ sample at a given rest-frame wavelength is thus contributed by multiple bands that observe galaxies at different redshifts. Therefore, any offset found in such a way is likely due to the inaccuracy of the SED templates instead of the systematic errors of our photometry, unless all the bands contributing to the rest-frame wavelength underestimate/overestimate fluxes in the same direction. We then subtract the offsets at all rest-frame wavelengths from our SED templates.

The zero-point offsets of all bands are shown in Table 4, both before and after applying the template correction. For most nonIRAC bands, the offset is $\sim 0.02 \mathrm{mag}$. The only non-IRAC band with significant offset is the ISAAC $K s$ band, whose offset is $0.06 \mathrm{mag}$. For IRAC 3.6, 4.5, and $5.8 \mu \mathrm{m}$, the offsets are reduced by the template correction to $\sim 0.05 \mathrm{mag}$. This value is consistent with the deviation between different measurements from our previous comparisons with other catalogs. The offset of IRAC $8.0 \mu \mathrm{m}$ is quite large, though. For galaxies at $z<0.4$ (about $20 \%$ of our spec- $z$ galaxies), this band is affected by the emissions of polycyclic aromatic hydrocarbon (PAH). The PAH emissions are not characterized in our SED models even after our correction, and thus the observed IRAC $8.0 \mu \mathrm{m}$ flux should be much brighter than the SED templates.

Overall, the systematic offset in our catalog is small $(\sim 0.02$ mag) for most UV to NIR bands and modest ( $\sim 0.05 \mathrm{mag})$ for ISAAC $K s$ and IRAC 3.6, 4.5, and 5.8 $\mu \mathrm{m}$. IRAC $8.0 \mu \mathrm{m}$ suffers from large systematic offsets, possibly due to PAH emissions. These derived zero-point offsets depend on both the redshift distribution of the spec- $z$ training sample and the choice of the photo- $z$ templates. Therefore, they can only serve as a rough evaluation of the quality of our catalog. We do not apply these offsets to our catalog.

\subsection{Photometric Redshifts}

We now measure photometric redshifts (photo-z's) of galaxies in our spectroscopic sample. We use the PEGASE v2.0 templates, both before and after applying our template correction to them. The accuracy of our photo- $z$ measurement is

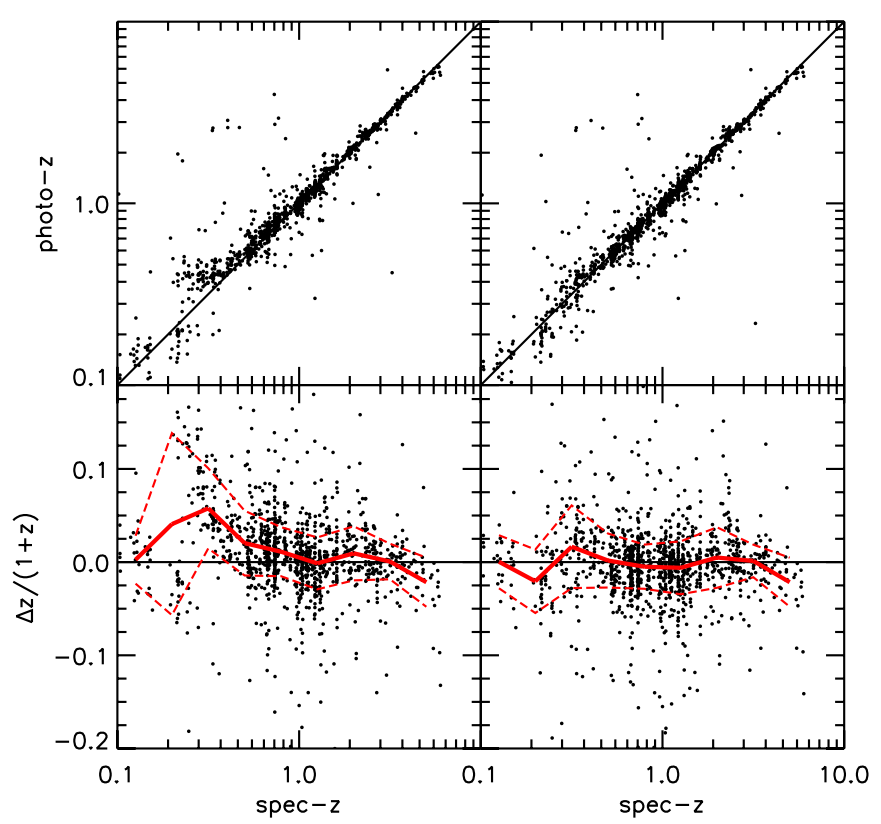

Figure 14. Accuracy of photometric redshifts measured with our catalog, before (left) and after (right) applying our template correction. In each column, the upper panel shows the comparison of photo- $z$ 's and spec-z's, and the lower panel shows the relative error of photo- $z$ as a function of spec- $z$. The solid and dashed lines in the lower panels stand for the mean and standard deviation (after $3 \sigma$ clipping) of the sample. Detailed statistics on the photo- $z$ accuracy are given in Table 5 .

(A color version of this figure is available in the online journal.)

shown in Figure 14, and detailed statistics on the accuracy are listed in Table 5. Our template correction mainly affects objects at $z<1$ and corrects the systematic overestimate of photo- $z$ among them. After the template correction, our measurement has the normalized median absolute deviation (NMAD; defined as $1.48 \times$ median $(|\Delta z| /(1+z)))$ of 0.028 and an outlier fraction (defined as $|\Delta z| /(1+z)>0.15)$ of $5.5 \%$ for all galaxies. These values are slightly better for bright $(H<24 \mathrm{AB})$ galaxies, but worse for faint $(H>24 \mathrm{AB})$ objects.

For galaxies at $z>1.5$, the accuracy increases to an NMAD $\sim 0.023$ and outlier fraction of $\sim 4.0 \%$, regardless of whether template correction is applied or not. The accuracy also depends little on the brightness of objects. This high accuracy indicates the importance of the CANDELS NIR data on photo- $z$ measurement for galaxies at intermediate or high redshift. The deep CANDELS photometry in our catalog effectively characterizes the Balmer/4000 $\AA$ break for galaxies at $z>1.5$ and hence yields an accurate photo- $z$ measurement for them. For low-redshift $(z<1.5)$ galaxies, their photo- $z$ 's are determined by both major spectral breaks (Lyman or Balmer) as well as the Rayleigh-Jeans regime of stellar emission. The latter is quantified by IRAC photometry in our catalog, which has larger uncertainty than CANDELS NIR photometry and hence reduces the accuracy of the photo- $z$ of $z<1.5$ galaxies.

It is also possible that the high accuracy of our photo- $z$ 's of $z>1.5$ galaxies is due to the narrow range of the spectral types of our spec- $z$ sample, which are fully covered by our SED-fitting templates. The narrow range of the spectral types could be physically real because galaxies at $z>1.5$ have a relatively simple star formation history, or it could be due to the selection bias of our spectroscopically observed sample. On the other hand, our templates may not fully cover the spectral types of $z<1.5$ galaxies because of their complicated star 
Table 5

Accuracy of Photometric Redshift

\begin{tabular}{|c|c|c|c|c|c|c|c|c|c|c|c|c|}
\hline & \multicolumn{4}{|c|}{ All } & \multicolumn{4}{|c|}{$z \leqslant 1.5$} & \multicolumn{4}{|c|}{$z>1.5$} \\
\hline & Mean & $\sigma$ & $f_{\text {outlier }}$ & NMAD & Mean & $\sigma$ & $f_{\text {outlier }}$ & NMAD & Mean & $\sigma$ & $f_{\text {outlier }}$ & NMAD \\
\hline All (before template correction) & 0.010 & 0.040 & $6.1 \%$ & 0.031 & 0.013 & 0.042 & $6.9 \%$ & 0.035 & 0.001 & 0.035 & $3.5 \%$ & 0.023 \\
\hline (after template correction) & -0.002 & 0.037 & $5.5 \%$ & 0.028 & -0.003 & 0.037 & $6.0 \%$ & 0.030 & -0.001 & 0.037 & $3.8 \%$ & 0.023 \\
\hline$H \leqslant 24 \mathrm{AB}$ (before template correction) & 0.012 & 0.041 & $5.3 \%$ & 0.032 & 0.013 & 0.042 & $5.8 \%$ & 0.034 & 0.004 & 0.037 & $3.0 \%$ & 0.023 \\
\hline (after template correction) & -0.002 & 0.037 & $4.8 \%$ & 0.028 & -0.003 & 0.037 & $5.1 \%$ & 0.029 & 0.002 & 0.039 & $3.6 \%$ & 0.024 \\
\hline$H>24 \mathrm{AB}$ (before template correction) & -0.001 & 0.039 & $11.7 \%$ & 0.029 & 0.009 & 0.050 & $22.2 \%$ & 0.057 & -0.006 & 0.031 & $4.4 \%$ & 0.022 \\
\hline (after template correction) & -0.004 & 0.036 & $10.3 \%$ & 0.027 & 0.001 & 0.043 & $19.0 \%$ & 0.046 & -0.008 & 0.030 & $4.4 \%$ & 0.021 \\
\hline
\end{tabular}

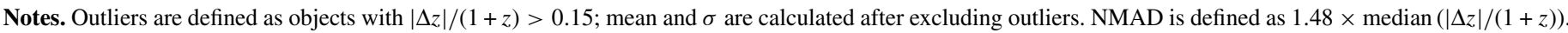

formation histories as well as variation in the dust extinction law, resulting in a decreased photo- $z$ accuracy. In order to improve the photo- $z$ at $z<1.5$, the uncertainties of SED-fitting templates in stellar evolutionary track, star formation history, dust extinction law, and spectral features should be taken into account. For example, Brammer et al. (2008) introduced a template error function to incorporate the above uncertainties together with the photometric uncertainty into the template-fitting algorithm. The template error function minimized systematic errors in their photo-z's.

Overall, our photo- $z$ measurement shows that our photometric catalog is sufficient to provide accurate photo- $z$ 's for galaxies, especially for those at $z>1.5$, where CANDELS NIR data greatly improve the quality of photo- $z$ 's. The CANDELS team is working on generating a photo- $z$ catalog for the GOODS-S field (T. Dahlen et al. 2013, in preparation).

\section{APPLICATION OF THE CATALOG TO STUDYING GALAXIES AT $z=2-4$}

\subsection{Detection Ability}

Because our catalog is constructed based on F160W detection, the depth and completeness of our F160W band place the primary limit on our detection ability. We use galaxy templates drawn from the updated version of Bruzual \& Charlot (2003; CB09) to predict the F160W photometry for different types of galaxies. The detection ability of our catalog is plotted in Figure 15 . For simplicity, we only discuss galaxies with stellar mass of $10^{10} M_{\odot}$ and age of $1 \mathrm{Gyr}$ at the epoch of observation. Galaxies with a constant star formation history and low dust extinction (e.g., $E(B-V)=0.15)$ can be detected with a completeness of $50 \%$ to $z \sim 3.4$ and $z \sim 4.6$ in our wide and deep regions, respectively. Due to their very red rest-frame UV and optical colors, star-forming galaxies with high dust extinction can only be detected with the same completeness to lower redshift. Galaxies with $E(B-V)=0.6$ can only be detected to the $50 \%$ completeness to $z \sim 2.0,2.4$ and 3.0 for the wide, deep, and HUDF regions, respectively. For passively evolving galaxies (PEGs), we can detect them with $50 \%$ completeness to $z \sim 2.8,3.2$, and 4.2 in the wide, deep, and HUDF regions, if they are unreddened. For galaxies with age older than $1 \mathrm{Gyr}$, the maximum detectable redshift is lower. For example, PEGs with age of 2 Gyr can be detected with a $50 \%$ completeness to $z=$ 2.4 and 2.8 for the wide and deep regions.

Figure 16 compares the differential number density of our catalog with that of other surveys that contain $H$-band data: GOODS NICMOS Survey (GNS, Conselice et al. 2011), GOODS-VLT Survey (Retzlaff et al. 2010), and NICMOS HDF Survey (Dickinson et al. 2000; Thompson 2003). The

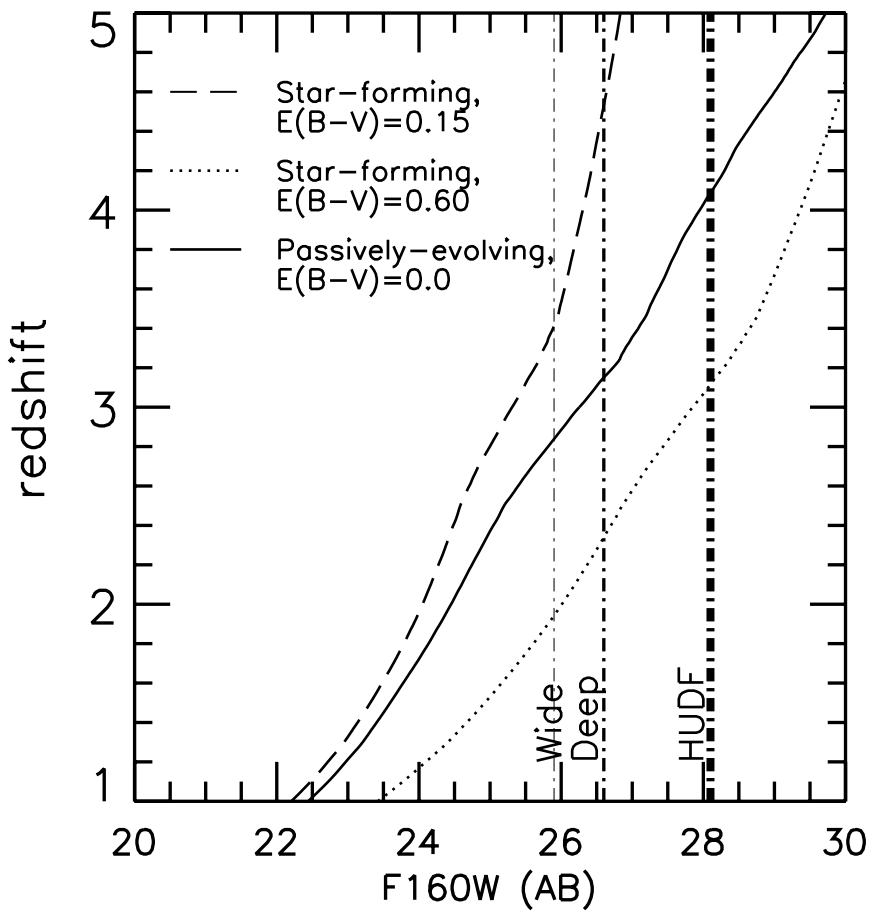

Figure 15. Detection ability of the CANDELS/GOODS-S catalog. Each curve shows the F160W magnitude of a model stellar population at different redshifts. All models have stellar mass of $10^{10} M_{\odot}$ and age of $1 \mathrm{Gyr}$ at the epoch of the observation. The solid curve denotes passively evolving galaxies that had a single burst of star formation and has no dust reddening. Dashed and dotted curves denote populations with a constant star formation history and different amounts of dust reddening characterized by $E(B-V)$ as indicated. Vertical lines show the representative $50 \%$ completeness levels in the three regions of the CANDELS/GOODS-S field.

representative 50\% completeness limits of GNS and GOODSVLT estimated through the best-fit power law are 25.1 and $24.3 \mathrm{AB}$ mag, respectively. Our wide (deep) region is 0.8 (1.5) and 1.6 (2.3) mag deeper than GNS and GOODS-VLT. Therefore, our catalog in the wide (deep) region is able to detect galaxies with stellar mass lower than the minimum detectable stellar mass of GNS and GOODS-VLT by a factor of two (four) and four (eight). The NICMOS HDF Survey is deeper than our deep region but fainter than our HUDF region. However, its small sky coverage, about $1 \operatorname{arcmin}^{2}$, brings large cosmic variance and small number statistics, as can be inferred from its irregular differential number density distribution.

To further demonstrate the detection ability of our catalog, we compare the overall photo- $z$ distributions of the three regions of our catalog to that of GM and FW in Figure 17. We cut each 


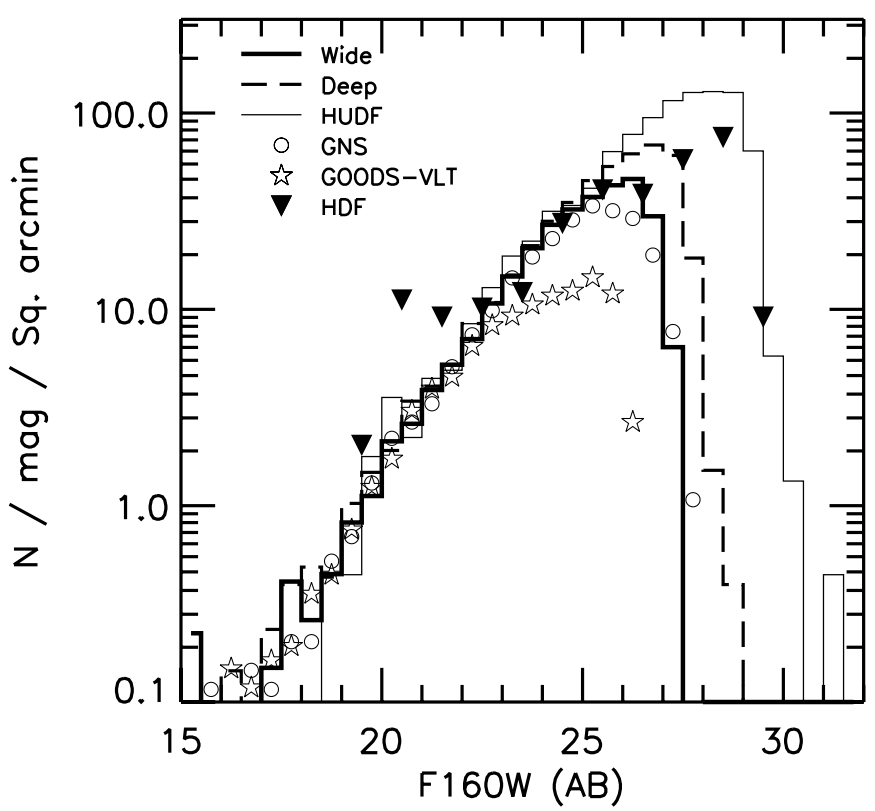

Figure 16. Differential number count comparison of $H$-band surveys. Lines show the three regions of our catalog. Open circles show GOODS NICMOS Survey (GNS; Conselice et al. 2011), stars show GOODS-VLT (Retzlaff et al. 2010), and triangles show NICMOS HDF Survey (Dickinson et al. 2000; Thompson 2003).

region/catalog to its $50 \%$ completeness magnitude through the best-fit power law to the differential source number density of each region/catalog, as discussed in Section 3.1. This threshold magnitude is $\mathrm{F} 850 \mathrm{LP}=26.4 \mathrm{AB}$ mag for $\mathrm{GM}$ and $K s=24.1$ for FW.

The photo- $z$ distributions of our wide region and GM are similar, suggesting a comparable detection ability at all redshifts, although our wide region has a marginal excess of detection ability at $z \sim 2$ and $z>5$. Our deep and HUDF regions surpass the GM catalog at all redshifts. The source number density in our deep (HUDF) region is higher than that of GM by a factor of $\sim 2(\sim 6)$ at $0<z<5$, and by a factor of $>4(>10)$ at $z>5$. All our three regions have much higher source number density in each redshift bin than FW.

\subsection{Balmer-break-selected Galaxies at $z \sim 2-4$}

Our deep NIR photometry provides an accurate description of the Balmer $/ 4000 \AA$ break for galaxies at $z \sim 2-4$, and enables selecting high-redshift galaxies through broadband colors that cover the break. Although current photo- $z$ measurements can achieve a fairly high accuracy, color selection methods still play an important role in selecting high-redshift galaxies. The bias, efficiency, and contamination of color selections can be explicitly determined through Monte Carlo simulation of selecting fake galaxies with various magnitude, size, color, and star formation history. Color selections are also fast and easy to reproduce.

A popular color-color selection criterion using the Balmer break is the $B z K$ method proposed by Daddi et al. (2004). This method, using the $(B-z)$ and $(z-K)$ colors to quantify the strength and curvature of the Balmer break, has been proven to be successful at selecting star-forming galaxies independent of their dust reddening as well as PEGs at $z \sim 2$. However, due to the lack of deep NIR photometry of large fields, extending this method to higher redshifts has not been extensively discussed. It was only extended to galaxies at $z \sim 3$ in the ERS field by

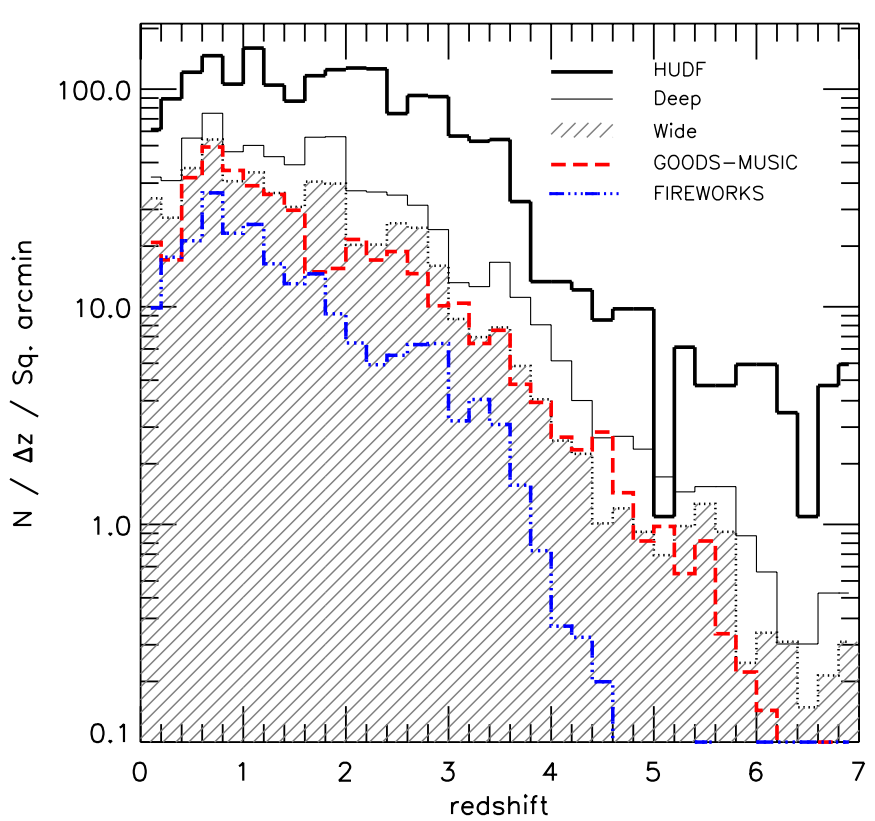

Figure 17. Photometric redshift distributions of the three regions of the CANDELS/GOODS-S catalog, GOODS-MUSIC, and FIREWORKS. Each catalog/region is cut to its $50 \%$ completeness threshold determined by the best-fit power-law method (see Section 3.1): F160W = 25.9, 26.6, 28.1 AB for the CANDELS wide, deep, and HUDF region; F850LP $=26.4 \mathrm{AB}$ for GOODS-MUSIC; and $K s=24.1 \mathrm{AB}$ for FIREWORKS.

(A color version of this figure is available in the online journal.)

Guo et al. (2012) using the $(V-J)$ and $(J-3.6 \mu \mathrm{m})$ colors. Our catalog with the deepest NIR photometry in the GOODS-S field is the first one that makes this Balmer break selection method practical in large fields. We apply the two selection criteria to our catalog and also extend them to $z \sim 4$.

The selection criteria used here are as follows.

1. Star-forming $B z K:(z-K s)>(B-z)-0.2$; and passively evolving $B z K:(z-K s) \leqslant(B-z)-0.2 \wedge(z-K s)>2.0$, where $B$ and $z$ are F435W and F850LP.

2. Star-forming VJL: $(J-L)>1.2 \times(V-J)$; and passively evolving VJL: $(J-L) \leqslant 1.2 \times(V-J) \wedge(J-L)>2.5$, where $V, J$, and $L$ are $\mathrm{F} 606 \mathrm{~W}, \mathrm{~F} 125 \mathrm{~W}$, and IRAC $3.6 \mu \mathrm{m}$.

3. Star-forming iHM: $(H-M)>1.375 \times(i-H)$; and passively evolving $i H M:(H-M) \leqslant 1.375 \times(i-H) \wedge$ $(H-M)>2.0$, where $i, H$, and $M$ are $\mathrm{F} 775 \mathrm{~W}, \mathrm{~F} 160 \mathrm{~W}$, and IRAC $4.5 \mu \mathrm{m}$.

For $B z K$, we demand $\mathrm{S} / \mathrm{N}>5$ in the $z$ and $K s$ bands. We use the ISAAC $K s$ band here because it covers the entire GOODS-S field. V. Sommariva et al. (2013, in preparation) are studying $B z K$ selected sources in both GOODS-S and UDS based on the HAWK-I $K s$ band. For $V J L$ and $i H M$, we demand $\mathrm{S} / \mathrm{N}>10$ in the two reddest bands used in each criterion.

The color-color diagram of each selection criterion is shown in Figure 18. The target redshift range of each criterion is $z \sim$ 1.5-2.5 for $B z K, 2.5-3.5$ for $V J L$, and 3.5-4.5 for $i H M$. Galaxies with photo- $z$ 's within each target range are overplotted in each diagram. These selection criteria do a fairly good job at selecting galaxies in their own target redshift range. Figure 19 shows the redshift distribution of selected galaxies in each criterion. We can roughly estimate the effectiveness and contamination of each criterion by comparing the redshift distribution of selected galaxies (blue histogram) and that of total galaxies (black histogram). The effectiveness, defined as the fraction of galaxies in the target redshift range that are selected by a 

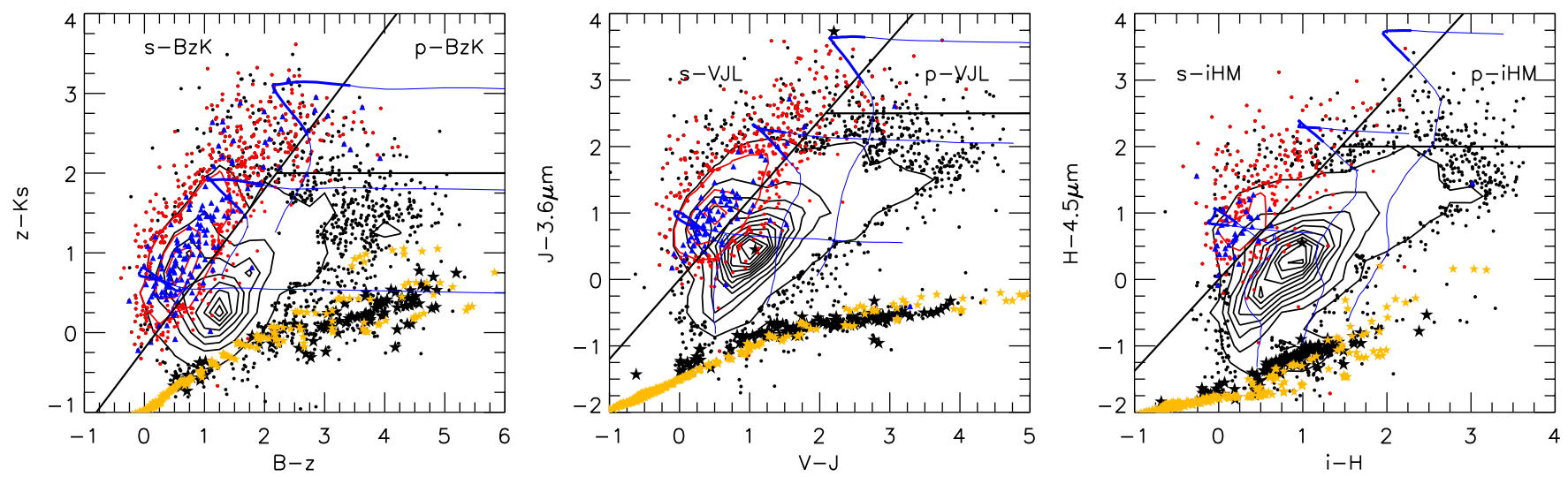

Figure 18. Color-color criteria of using the Balmer break to select galaxies at $z \sim 2$ (BzK, left), $z \sim 3$ (VJL, middle), and $z \sim 4$ (iHM, right). Windows for selecting star-forming (s-) and passively evolving galaxies (p-) are separated by black solid lines and labeled. In each panel, black contours and dots show the distribution of all galaxies in our catalog with high S/Ns ( $>5$ for $z$ and $K s$ in $B z K,>10$ for $J$ and $3.6 \mu \mathrm{m}$ in $V J L$, and $>10$ for $H$ and $4.5 \mu \mathrm{m}$ in $i H M$ ). Red contours and dots show the distribution of galaxies with photo-z's within the redshift range of interest $([1.5,2.5]$ for $B z K,[2.5,3.5]$ for $V J L$, and $[3.5,4.5]$ for $i H M)$. Blue triangles are galaxies with spec-z's within the redshift range of interest of each criterion. Blue curves show the track of star-forming galaxies with constant star formation history, age of $1 \mathrm{Gyr}$, and reddening $E(B-V)=0.0,0.3$, and 0.6 (for top to bottom in each panel). In each curve, the thick region shows the redshift range of interest. Black stars show objects with the F160W SExtractor parameter STAR_CLASS $>0.98$. Yellow stars show stars in the synthetic library of Lejeune et al. (1997).

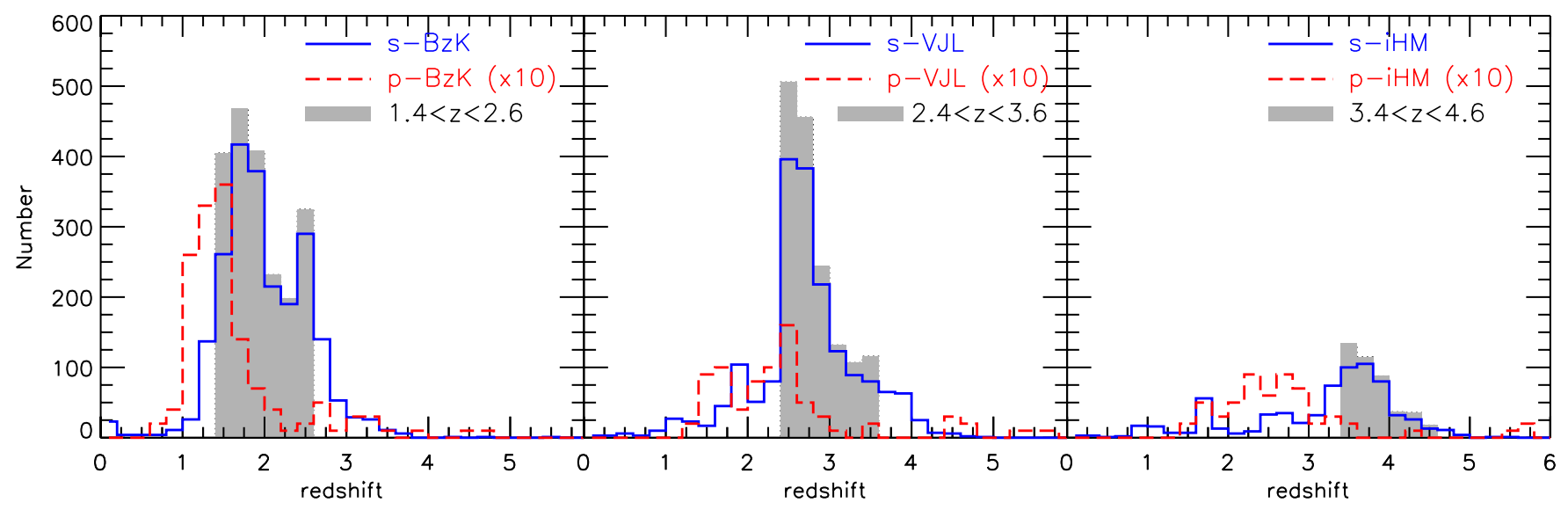

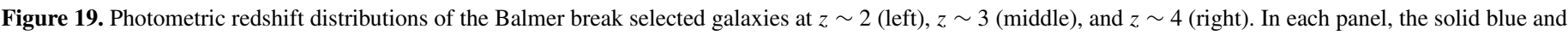

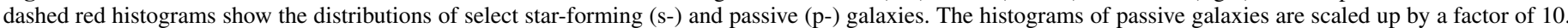

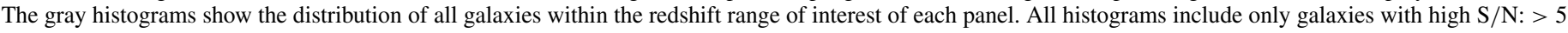
for $z$ and $K s$ in the left panel, $>10$ for $J$ and $3.6 \mu \mathrm{m}$ middle, and $>10$ for $H$ and $4.5 \mu \mathrm{m}$ right.

given criterion, is about $86 \%, 83 \%$, and $83 \%$ for $B z K, V J L$, and $i H M$, respectively. The contamination, defined as the fraction of selected galaxies that are not within the target redshift range of the selection criterion, is about $21 \%, 30 \%$, and $50 \%$ for $B z K$, $V J L$, and $i H M$, respectively.

We also compare the observed and synthetic stellar locus in the color-color diagrams to examine our NIR photometry. Since our tests on the ACS-WFC3 colors of stars show no offset between the observed and synthetic stars (Section 5.1), any deviation between the observed and synthetic stars in these diagrams is caused by the systematic offset of the NIR bands. In the $B z K$ diagram, the colors of observed stars show good agreement with those of stars in the synthetic library of Lejeune et al. (1997), suggesting no systematic offset in our ISAAC $K s$-band photometry. In the $V J L$ and $i H M$ diagrams, a mild deviation about $\sim 0.05 \mathrm{mag}$ is found between the observed and synthetic stars. This deviation may indicate that our 3.6 and $4.5 \mu \mathrm{m}$ fluxes are overestimated by $\sim 0.05 \mathrm{mag}$. It can also be due to, however, the inaccurate calibration of the NIR regime of the stellar libraries.

PEGs at $z>3$ potentially hold important clues to the processes that quench star formation in massive galaxies, and to the emergence of the Hubble sequence. As discussed above, our catalog is able to detect such galaxies to $z>3$. Although the redshift distribution of selected passive galaxies (red histograms in Figure 19) declines dramatically at $z>3$, we still find 26 galaxies from the passive selection windows. The major contamination source in the passive selection windows is dusty star-forming galaxies at lower redshift. In order to exclude the contamination, we remove 13 sources with MIPS $24 \mu \mathrm{m}$ detection. If the remaining 13 galaxies are confirmed to have passive nature, their star formation activity should have ceased at least $0.5-1$ Gyr ago, i.e., at $z \sim 5$.

\subsection{Red Galaxies at $z \sim 2-4$}

Based on their positions in the color-magnitude diagram (CMD), local galaxies can be roughly divided into two populations: blue cloud and red sequence (e.g., Strateva et al. 2001; Blanton et al. 2003; Baldry et al. 2004). Galaxies in the blue cloud are reliably classified as spiral galaxies with ongoing star formation, and their color is strongly related to the recent star formation history. Galaxies in the red sequence (except dustreddened spirials) generally have little recent star formation. 


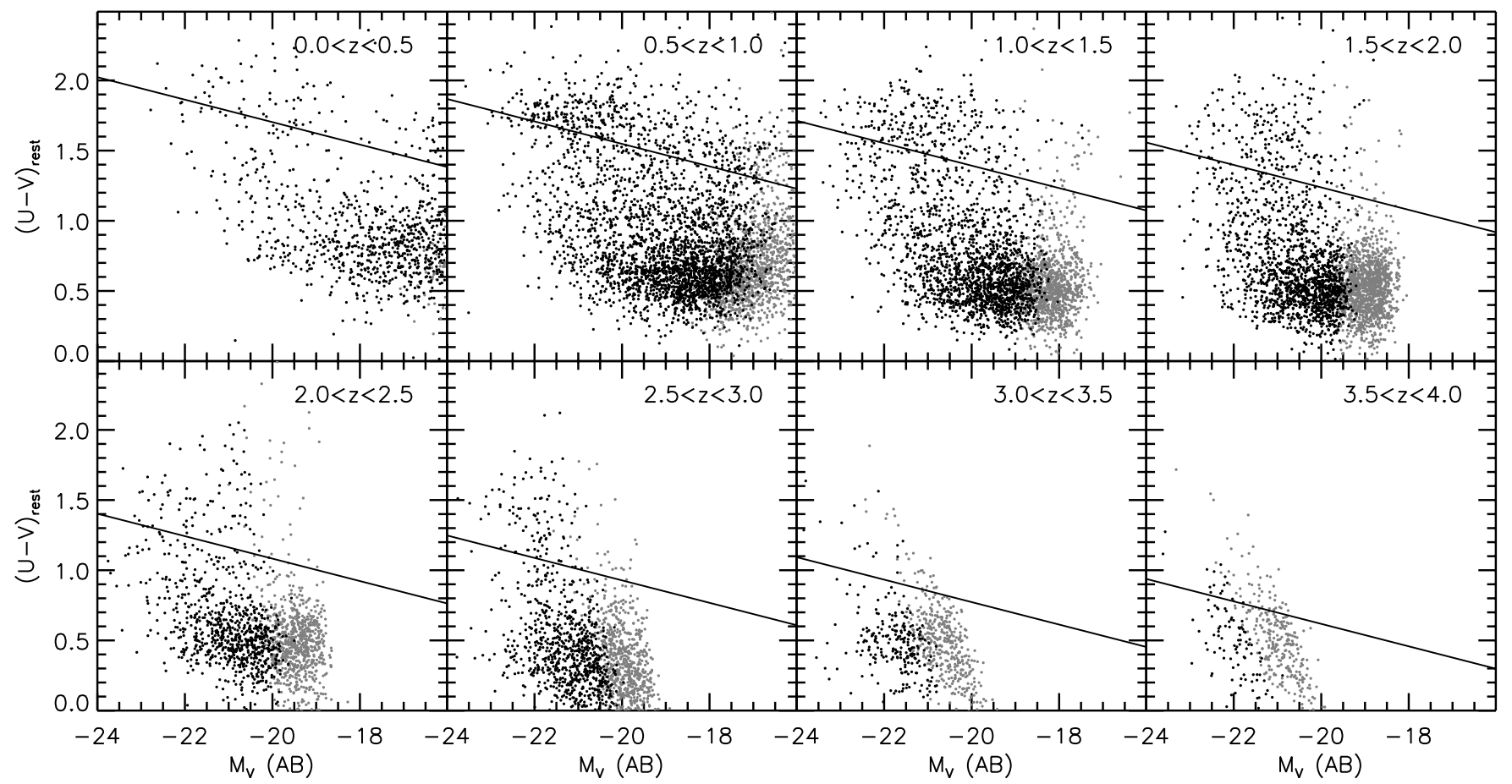

Figure 20. Rest-frame color-magnitude diagram of galaxies in the CANDELS/GOODS-S catalog. Black points are galaxies with F160W $<24.9$ AB (50\% completeness level of the wide region) and gray points are galaxies with $24.9<\mathrm{F} 160 \mathrm{~W}<25.9 \mathrm{AB}$ (50\% completeness level of the deep region). The black line in each panel is the extrapolation of the red-blue separation of Bell et al. (2004) to the redshift of the panel.

This color bimodality has been reported up to $z \sim 3$ (e.g., Bell et al. 2004; Cassata et al. 2008; Kriek et al. 2009; Brammer et al. 2009; Whitaker et al. 2011). It is unclear, however, at which cosmic epoch this color bimodality emerges, owing to limitations of the accuracy of photo-z's and rest-frame UV-optical colors.

The deep NIR photometry of our catalog provides a unique tool to detect red galaxies at $z>3$ to investigate the origin of the color bimodality. Figure 20 shows the rest-frame CMD of galaxies at different redshifts in our catalog. We use the extrapolation of the red-blue separation line of Bell et al. (2004) to separate the blue cloud and red sequence. The red sequence is clearly shown at $z<2$. At $2<z<3$, the red sequence becomes less obvious, but there is still a large amount of galaxies which are well separated from the blue cloud and enter the red region. At $z>3$, due to the decline in the number of galaxies, the separation of the two populations is not as clear as at lower redshifts. However, a handful of galaxies still have red enough rest-frame UV-optical color to enter the red region, suggesting the existence of passive galaxies at $z>3$.

We present a few examples of red and blue SEDs at $3<z<4$ in our catalog in Figure 21 to show the quality of our photometry. The small uncertainties on the rest-frame UV-optical colors enable reliable separation of different types of SEDs of galaxies up to $z \sim 4$. However, although red galaxies at $z \sim 4$ suggest the existence of evolved galaxies with little ongoing star formation at such high redshift, they could also be dusty star-forming galaxies whose rest-frame UV-optical colors are reddened by dust. To investigate the nature, stellar masses, and number densities of these red galaxies is a subject for future works.

\section{SUMMARY}

We present a UV to mid-infrared multi-wavelength catalog in the CANDELS/GOODS-S region, combining the newly obtained CANDELS HST/WFC3 F105W, F125W, and F160W data with existing public data. The catalog is based on source detection in the WFC3 F160W band. To maximize the scientific yields of our catalog, we construct a "max-depth" F160W mosaic, which includes data from the CANDELS deep and wide observations as well as two previous HST/WFC3 programs, ERS and HUDF09. The F160W mosaic, providing the deepest photometry for each individual source in GOODS-S, reaches a $5 \sigma$ limiting depth (within an aperture of $0{ }^{\prime \prime} 17$, the FWHM of the F160W) of 27.4, 28.2, and 29.7 AB mag for CANDELS wide, deep + ERS, and HUDF regions, respectively. The representative $50 \%$ completeness of our catalog reaches $25.9,26.6$, and 28.1 AB mag in the F160W band for the three regions. The catalog contains 34,930 sources in total.

In addition to WFC3 NIR bands, the catalog also includes data from UV ( $U$ band from both CTIO/MOSAIC and VLT/VIMOS), optical (GOODS HST/ACS F435W, F606W, F775W, F814W, and F850LP), and IR (ERS WFC3 F098M, VLT/ISAAC $K s$, VLT/HAWK-I $K s$, and Spitzer/IRAC 3.6, $4.5,5.8,8.0 \mu \mathrm{m})$ observations. We use SExtractor to measure photometry for all available $H S T$ bands from PSF-matched images. For other low-resolution bands, whose FWHMs of PSFs vary by almost a factor of 10 , we use our profile template-fitting package, TFIT, to measure the uniform photometry among them.

The quality of the multi-wavelength catalog is thoroughly tested. First, the comparison between the ACS-WFC3 colors of stars in our catalog and those of stars in synthetic stellar libraries shows excellent agreements. Second, the photometry difference between our catalog and GM and FW is nearly zero over the magnitude range to $\sim 24 \mathrm{AB}$ mag, with the worst offset being $\sim 0.05 \mathrm{mag}$. We discuss the possible reasons of the deviations between our and other catalogs at the faint end. Last, the zero-point offsets measured by comparing the observed SEDs to the best-fit stellar population synthesis templates of spectroscopically observed galaxies are $\lesssim 0.02 \mathrm{mag}$ for most UV to NIR bands. The photo- $z$ 's measured with our catalog can reach the accuracy of NMAD $\sim 0.028$. 


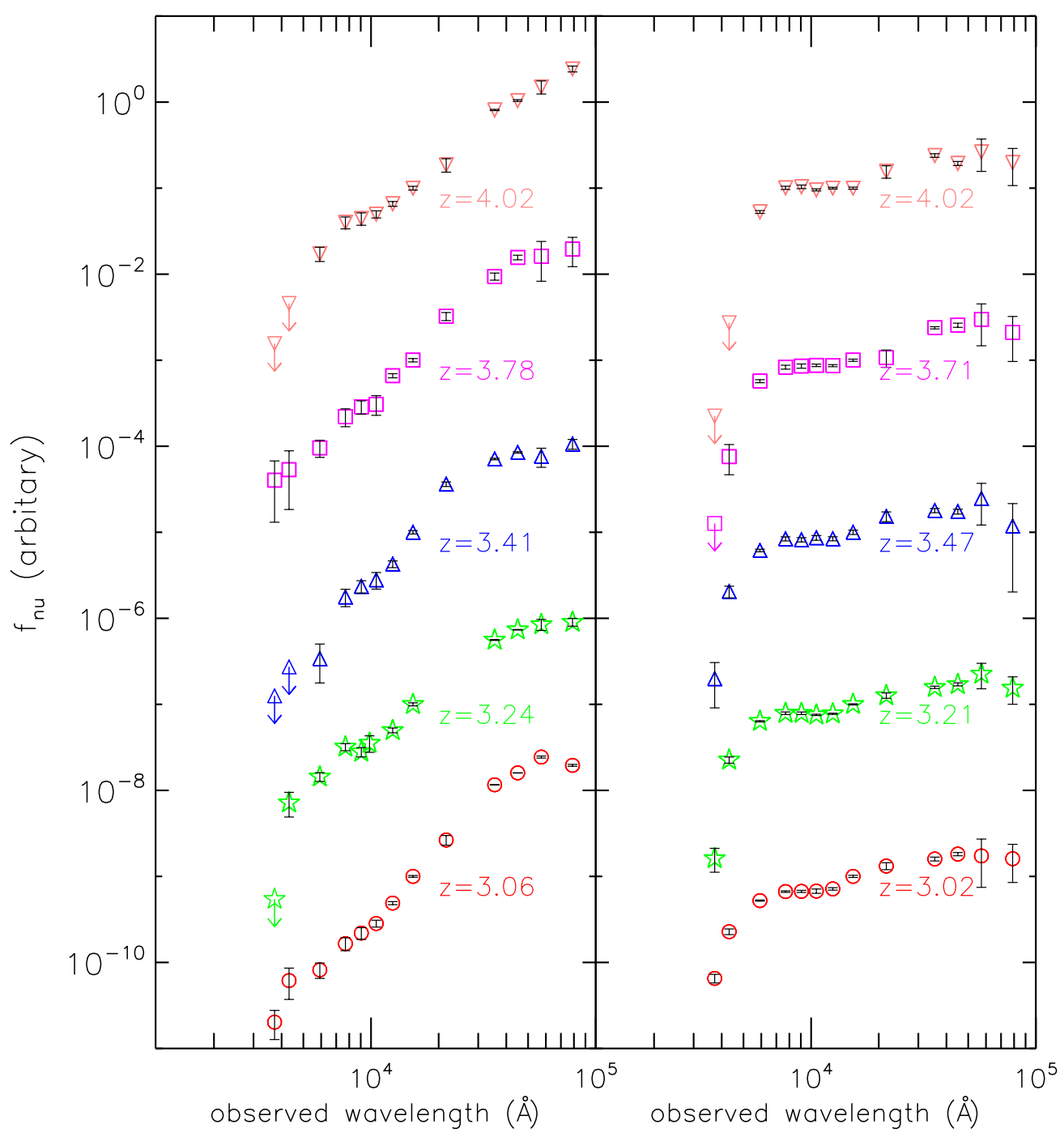

Figure 21. Examples of SEDs of red (left column) and blue (right column) galaxies at $3<z<4$ in the CANDELS/GOODS-S catalog. The redshifts of the galaxies increase from the bottom to the top, as shown by labels.

(A color version of this figure is available in the online journal.)

Our catalog is able to detect star-forming galaxies with stellar mass of $10^{10} M_{\odot}$ to $z \sim 3.4,4.6$, and 8.0 at a $50 \%$ completeness in the wide, deep, and ultra-deep regions, respectively. Passive galaxies with the same stellar mass can be detected at a 50\% completeness to $z \sim 2.8,3.2$, and 4.2 in the above regions. Our catalog in the wide and deep regions is about 1-2 mag deeper than other public $H$-band surveys. The minimum detectable stellar mass in the wide and deep regions of our catalog is therefore smaller than that of other $H$-band surveys by a factor of about 3-6.

To provide an example of applying our catalog to study distant galaxies, we use color criteria to select both star-forming and passively evolving galaxies at $z=2-4$ through the strength and curvature of the Balmer Break. We examine the redshift distribution of selected galaxies and discuss the effectiveness and contamination of the selection criteria. The detection of red galaxies with an obvious Balmer break in our catalog suggests the existence of passive galaxies at $z>3$.

We also study the evolution of the rest-frame CMD, $(U-V)$ versus $M_{V}$, from $z=0$ to $z=4$ to investigate the emergence of the color bimodality in the universe. A handful of red galaxies, possibly a combination of passive galaxies and dusty starforming galaxies, are detected in our catalog at $z \sim 4$.
The CANDELS GOODS-S multi-wavelength catalog and its associated files are publicly available on the CANDELS Web site, in the MAST, via the online version of this journal, and the CDS, as well as in the Rainbow Database.

We thank the anonymous referee for constructive comments that improve this article. Support for program number HSTGO-12060 was provided by NASA through a grant from the Space Telescope Science Institute, which is operated by the Association of Universities for Research in Astronomy, Incorporated, under NASA contract NAS5-26555. Y.G. and the authors from UCSC acknowledge support from NASA HST grant GO-12060.10-A and NSF grant AST-0808133. J.S.D. acknowledges the support of the European Research Council via the award of an Advanced Grant and the support of the Royal Society via a Wolfson Research Merit Award. This work is based in part on observations made with the Spitzer Space Telescope, which is operated by the Jet Propulsion Laboratory, California Institute of Technology, under a contract with NASA. Support for this work was provided by NASA through an award issued by JPL/Caltech.

Facilities: HST (ACS, WFC3), Spitzer (IRAC) 


\section{REFERENCES}

Ashby, M. L. N., Willner, S. P., Fazio, G. G., et al. 2013, ApJ, 769, 80 Baldry, I. K., Glazebrook, K., Brinkmann, J., et al. 2004, ApJ, 600, 681 Balestra, I., Mainieri, V., Popesso, P., et al. 2010, A\&A, 512, A12

Barden, M., Häußler, B., Peng, C. Y., McIntosh, D. H., \& Guo, Y. 2012, MNRAS, 422, 449

Barro, G., Pérez-González, P. G., Gallego, J., et al. 2011, ApJS, 193, 13

Bell, E. F., Wolf, C., Meisenheimer, K., et al. 2004, ApJ, 608, 752

Blanton, M. R., Hogg, D. W., Bahcall, N. A., et al. 2003, ApJ, 594, 186

Bouwens, R. J., Illingworth, G. D., Franx, M., et al. 2009, ApJ, 705, 936

Bouwens, R. J., Illingworth, G. D., Oesch, P. A., et al. 2010a, ApJL, 709, L133

Bouwens, R. J., Illingworth, G. D., Oesch, P. A., et al. 2010b, ApJL, 708, L69

Bouwens, R. J., Illingworth, G. D., Oesch, P. A., et al. 2012, ApJL, 752, L5

Brammer, G. B., van Dokkum, P. G., \& Coppi, P. 2008, ApJ, 686, 1503

Brammer, G. B., Whitaker, K. E., van Dokkum, P. G., et al. 2009, ApJL, 706, L173

Bruzual, G., \& Charlot, S. 2003, MNRAS, 344, 1000

Casertano, S., de Mello, D., Dickinson, M., et al. 2000, AJ, 120, 2747

Cassata, P., Cimatti, A., Kurk, J., et al. 2008, A\&A, 483, L39

Cimatti, A., Cassata, P., Pozzetti, L., et al. 2008, A\&A, 482, 21

Coe, D., Zitrin, A., Carrasco, M., et al. 2013, ApJ, 762, 32

Conselice, C. J., Bluck, A. F. L., Buitrago, F., et al. 2011, MNRAS, 413, 80

Daddi, E., Cimatti, A., Renzini, A., et al. 2004, ApJ, 617, 746

Daddi, E., Dickinson, M., Morrison, G., et al. 2007, ApJ, 670, 156

Dahlen, T., Mobasher, B., Dickinson, M., et al. 2010, ApJ, 724, 425

Dickinson, M., Hanley, C., Elston, R., et al. 2000, ApJ, 531, 624

Dunlop, J. S., McLure, R. J., Robertson, B. E., et al. 2012, MNRAS, 420, 901

Fazio, G. G., Hora, J. L., Allen, L. E., et al. 2004, ApJS, 154, 10

Finkelstein, S. L., Papovich, C., Giavalisco, M., et al. 2010, ApJ, 719, 1250

Finkelstein, S. L., Papovich, C., Ryan, R. E., et al. 2012a, ApJ, 758, 93

Finkelstein, S. L., Papovich, C., Salmon, B., et al. 2012b, ApJ, 756, 164

Fioc, M., \& Rocca-Volmerange, B. 1997, A\&A, 326, 950

Fontana, A., Vanzella, E., Pentericci, L., et al. 2010, ApJL, 725, L205

Galametz, A., Grazian, A., Fontana, A., et al. 2013, ApJS, 206, 10

Giacconi, R., Zirm, A., Wang, J., et al. 2002, ApJS, 139, 369

Giavalisco, M., Ferguson, H. C., Koekemoer, A. M., et al. 2004, ApJL, 600, L93

Gray, M. E., Wolf, C., Barden, M., et al. 2009, MNRAS, 393, 1275

Grazian, A., Castellano, M., Koekemoer, A. M., et al. 2011, A\&A, 532, A33

Grazian, A., Fontana, A., de Santis, C., et al. 2006, A\&A, 449, 951

Grogin, N. A., Kocevski, D. D., Faber, S. M., et al. 2011, ApJS, 197, 35

Guo, Y., Giavalisco, M., Cassata, P., et al. 2012, ApJ, 749, 149

Hopkins, A. M. 2004, ApJ, 615, 209

Hopkins, A. M., \& Beacom, J. F. 2006, ApJ, 651, 142

Kellermann, K. I., Fomalont, E. B., Mainieri, V., et al. 2008, ApJS, 179, 71
Koekemoer, A. M., Faber, S. M., Ferguson, H. C., et al. 2011, ApJS, 197, 36

Kriek, M., van Dokkum, P. G., Franx, M., Illingworth, G. D., \& Magee, D. K. 2009, ApJL, 705, L71

Labbé, I., Bouwens, R., Illingworth, G. D., \& Franx, M. 2006, ApJL, 649, L67

Laidler, V. G., Papovich, C., Grogin, N. A., et al. 2007, PASP, 119, 1325

Le Fèvre, O., Vettolani, G., Paltani, S., et al. 2004, A\&A, 428, 1043

Lee, K.-S., Ferguson, H. C., Wiklind, T., et al. 2012, ApJ, 752, 66

Lejeune, T., Cuisinier, F., \& Buser, R. 1997, A\&AS, 125, 229

Lutz, D., Poglitsch, A., Altieri, B., et al. 2011, A\&A, 532, A90

McLure, R. J., Dunlop, J. S., Cirasuolo, M., et al. 2010, MNRAS, 403, 960

Middelberg, E., Deller, A., Morgan, J., et al. 2011, A\&A, 526, A74

Mignoli, M., Cimatti, A., Zamorani, G., et al. 2005, A\&A, 437, 883

Nonino, M., Dickinson, M., Rosati, P., et al. 2009, ApJS, 183, 244

Norris, R. P., Afonso, J., Appleton, P. N., et al. 2006, AJ, 132, 2409

Oesch, P. A., Bouwens, R. J., Illingworth, G. D., et al. 2010, ApJL, 709, L16

Oke, J. B. 1974, ApJS, 27, 21

Oliver, S. J., Bock, J., Altieri, B., et al. 2012, MNRAS, 424, 1614

Pérez-González, P. G., Rieke, G. H., Villar, V., et al. 2008, ApJ, 675, 234

Popesso, P., Dickinson, M., Nonino, M., et al. 2009, A\&A, 494, 443

Reddy, N. A., \& Steidel, C. C. 2009, ApJ, 692, 778

Reddy, N. A., Steidel, C. C., Pettini, M., et al. 2008, ApJS, 175, 48

Retzlaff, J., Rosati, P., Dickinson, M., et al. 2010, A\&A, 511, A50

Riess, A. G., Strolger, L.-G., Casertano, S., et al. 2007, ApJ, 659, 98

Rix, H.-W., Barden, M., Beckwith, S. V. W., et al. 2004, ApJS, 152, 163

Robin, A. C., Reylé, C., Derrière, S., \& Picaud, S. 2003, A\&A, 409, 523

Santini, P., Fontana, A., Grazian, A., et al. 2009, A\&A, 504, 751

Scott, K. S., Yun, M. S., Wilson, G. W., et al. 2010, MNRAS, 405, 2260

Silverman, J. D., Mainieri, V., Salvato, M., et al. 2010, ApJS, 191, 124

Smith, J. A., Tucker, D. L., Kent, S., et al. 2002, AJ, 123, 2121

Strateva, I., Ivezić, Ž., Knapp, G. R., et al. 2001, AJ, 122, 1861

Szokoly, G. P., Bergeron, J., Hasinger, G., et al. 2004, ApJS, 155, 271

Thompson, R. I. 2003, ApJ, 596, 748

Tokunaga, A. T., \& Vacca, W. D. 2005, PASP, 117, 421

Trenti, M., Bradley, L. D., Stiavelli, M., et al. 2011, ApJL, 727, L39

van der Wel, A., Bell, E. F., Häussler, B., et al. 2012, ApJS, 203, 24

Vanzella, E., Cristiani, S., Dickinson, M., et al. 2008, A\&A, 478, 83

Wang, T., Huang, J.-S., Faber, S. M., et al. 2012, ApJ, 752, 134

Wang, W.-H., Cowie, L. L., Barger, A. J., Keenan, R. C., \& Ting, H.-C. 2010, ApJS, 187, 251

Whitaker, K. E., Labbé, I., van Dokkum, P. G., et al. 2011, ApJ, 735, 86

Windhorst, R. A., Cohen, S. H., Hathi, N. P., et al. 2011, ApJS, 193, 27

Wuyts, S., Labbé, I., Schreiber, N. M. F., et al. 2008, ApJ, 682, 985

Xue, Y. Q., Luo, B., Brandt, W. N., et al. 2011, ApJS, 195, 10

Yan, H., Finkelstein, S. L., Huang, K.-H., et al. 2012, ApJ, 761, 177

Zinn, P.-C., Middelberg, E., Norris, R. P., et al. 2012, A\&A, 544, A38 